\title{
Commercial Building Motor Protection Response Report
}

\author{
Dan James
}

John Kueck

June 2015

Special thanks to the subject matter expert Independent review participants:

Avtar Gill: with Johnson Controls

Michael Gust: with Incontrol

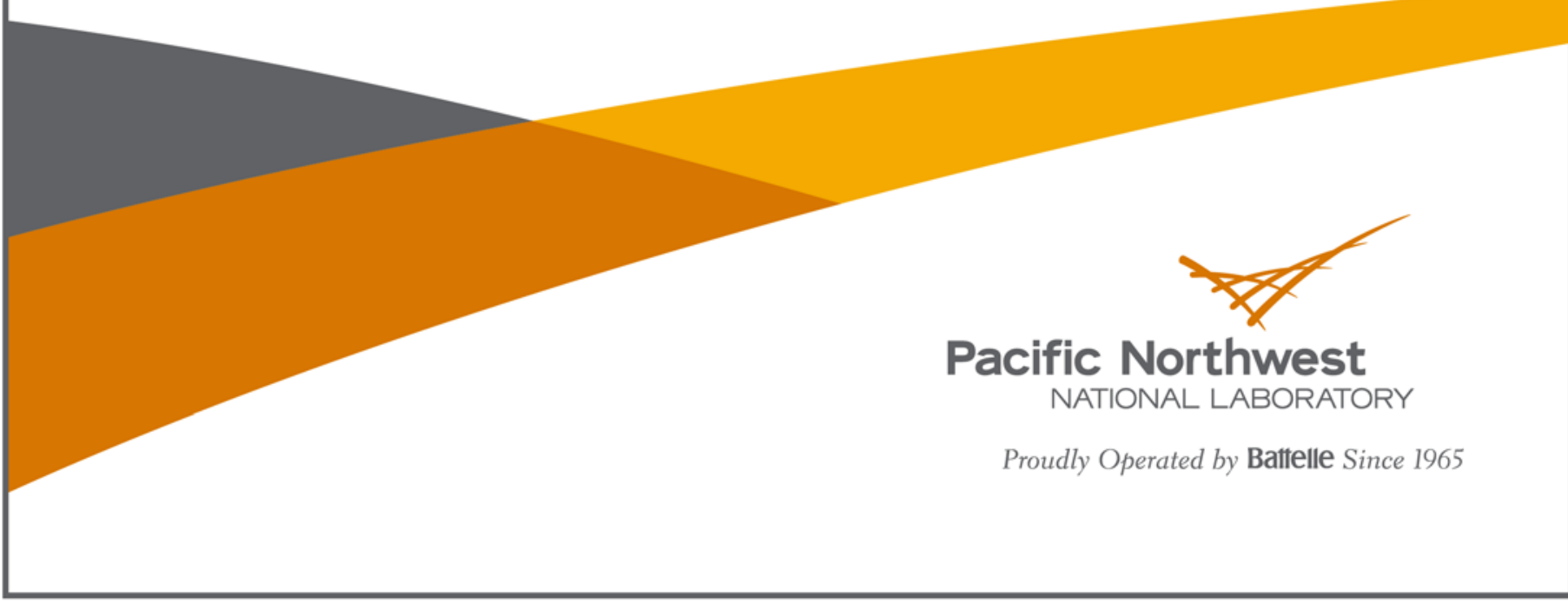




\title{
DISCLAIMER
}

This report was prepared as an account of work sponsored by an agency of the United States Government. Neither the United States Government nor any agency thereof, nor Battelle Memorial Institute, nor any of their employees, makes any warranty, express or implied, or assumes any legal liability or responsibility for the accuracy, completeness, or usefulness of any information, apparatus, product, or process disclosed, or represents that its use would not infringe privately owned rights. Reference herein to any specific commercial product, process, or service by trade name, trademark, manufacturer, or otherwise does not necessarily constitute or imply its endorsement, recommendation, or favoring by the United States Government or any agency thereof, or Battelle Memorial Institute. The views and opinions of authors expressed herein do not necessarily state or reflect those of the United States Government or any agency thereof.

\author{
PACIFIC NORTHWEST NATIONAL LABORATORY \\ operated by \\ BATTELLE \\ for the \\ UNITED STATES DEPARTMENT OF ENERGY \\ under Contract DE-AC05-76RL01830
}

Printed in the United States of America

$$
\begin{aligned}
& \text { Available to DOE and DOE contractors from the } \\
& \text { Office of Scientific and Technical Information, } \\
& \text { P.O. Box } 62 \text {, Oak Ridge, TN } 37831-0062 \text {; } \\
& \text { ph: }(865) 576-8401 \\
& \text { fax: }(865) 576-5728 \\
& \text { email: reports@adonis.osti.gov }
\end{aligned}
$$

Available to the public from the National Technical Information Service 5301 Shawnee Rd., Alexandria, VA 22312

ph: (800) 553-NTIS (6847)

email: orders@intis.gov <http://www.ntis.gov/about/form.aspx>

Online ordering: http:/www.ntis.gov 


\section{Motor Response and Protection in Commercial Buildings}

\section{Introduction}

A Fault Induced Delayed Voltage Recovery (FIDVR) event is the phenomenon whereby system voltage remains at significantly reduced levels for several seconds after a transmission, sub-transmission, or distribution fault has been cleared. The effect is thought to be caused by the stalling of highly concentrated induction motor loads with constant torque. These motors stall in response to low voltage. The stalled motors draw more reactive power from the grid, and that holds down the local voltage. A vicious circle is created. After several seconds of being stalled, motor protection devices begin to act and trip the motors to prevent them from overheating. As a result, there will be a large decrease in the load on the power system, with a potential secondary effect of high system voltage. That response is particularly likely if the protection response is slower than the voltage regulation response of the system.

The project described in this report is the result of a multi-year effort by the U.S. Department of Energy to assist the Western Electricity Coordinating Council's (WECC's) Load Modeling Task Force (LMTF) as they attempt to address these modeling challenges. This is one of many tasks funded under the American Recovery and Reinvestment Act (ARRA) Interconnection Planning activities; this report updates Pacific Northwest National Laboratory's (PNNL's) progress on the motor response and protection in commercial buildings. PNNL developed building motor response tables for a given set of commercial building types with voltage variances and time frames of interest. PNNL categorized motor protection and control responses for these voltage variances and times along with energy management system control logic restart times and motor protection trip delays. PNNL identified "make and break" times of relays and contactors for the given voltage variances and recovery times. The results are shown in a set of tables giving our estimated motor response for the type of motor load, type of building, and level and duration of the voltage variance.

\section{Conclusion}

While motor protection schemas are quite standard for motors in specific manufacturers' equipment and for specific motor applications, there are many variables to determine when motors will drop off line and reenergize on certain FIDVR events. There are just as many factors for determining what the true motor load is in a particular type of building at a given point in time. Because of the vast amount of variables that affect motor response at the time of a FIDVR event, motor protection response in this report is referred to in general terms and cannot be definitively nailed down in all certainty. By disregarding the motors and equipment that skew the average and by widening the timing ranges and percentages, most motors can be predicted to drop offline in certain ranges and reenergize within a certain time period. Although this is not accurate for all motors, this method will capture the bulk of the motors and their response to different lengths and depths of FIDR events. This report along with the tables in Appendix A provides details on voltage responses of typical motor loads found in commercial buildings. Depending on the magnitude and duration of the voltage sag, motors may ride through, trip, or stall. When voltages recover, motors may immediately reenergize and restart, or delay for a few minutes, or stay stalled. The estimated motor response is given for both the voltage sag magnitude and voltage sag duration. These response estimates are based on experience and available test data. Good data is available for voltage sag response for many components such as relays and contactors, but little data is available for both voltage sag and recovery response. The tables in Appendix A include data from recent voltage sag and recovery tests performed by Southern California Edison (SCE) and Bonneville Power Administration (BPA) on air conditioners and energy management systems. The response of the motor can vary greatly depending on the type of 
protection and control. The time duration for the voltage sag consists of those times that are of interest for bulk power system modelers. The times of interest are 5, 10, and 20 cycles, 2 seconds, and 3 minutes.

\section{Table Breakdown}

In the Appendix A, tables are prepared in two categories of voltage drop: voltage drop that ranges from $75 \%$ to $100 \%$ of nominal and drop that ranges from $50 \%$ to $75 \%$ of nominal. In general, most motor control equipment will ride through sags down to $75 \%$. In some cases, for larger motors, under voltage protection will trip motors for sags to $80 \%$ of nominal for 2 seconds.

The types of commercial buildings that have been considered are as follows:

- Food Service (Fast Food) (McDonalds)

- Supermarket (Albertson's)

- Other (Hotels, Residential Care)

- Office

- $\quad 20 \mathrm{k}-100 \mathrm{k}$ sf office building motor response (small office)

- $\quad 100 \mathrm{k}-1 \mathrm{~m}$ sf office building motor response (large office)

- Retail (Both Big and Small, by square feet)

- $\quad 5 \mathrm{k}-15 \mathrm{k}$ sf retail building motor table (service station)

- $\quad 15 \mathrm{k}-40 \mathrm{k}$ sf retail building motor response (strip mall)

- $\quad 40 \mathrm{k}-100 \mathrm{k}$ sf retail building motor response (big box stores)

- Warehouse

The tables in Appendix A provide columns for equipment, motors, protection, and controls. This gives the reader an understanding of the type of motor, what equipment it is used in, how it is usually protected, and what type of control system is normally used.

The commercial office building tables are broken down by square foot to indicate which of the following apply:

- Larger, high-rise office buildings usually have an energy management system (EMS) that senses outdoor temperature, planned building occupancies, day of week, etc., to operate the chillers, boilers, fans, and pumps most efficiently. The mechanical engineer and architect dictate the complexity of the mechanical systems that are present.

- Larger office buildings have variable air volume or VAV systems that also modulate the conditioned air flow throughout the building to optimize efficiency. VAV systems generally have some type of building automation system or BAS, the more complex the HVAC system the more it benefits from advanced control. Sequencing of the air handlers and optimizing that with the heating/cooling needed from the chillers and boilers sometimes makes for a complex control system. The more different mechanical systems that need to be sequenced together the more complex things can become. Small office buildings can sometimes get away with very simple thermostat controls and time clocks; in these cases, a computerized control system may not be used. 
- The tables have a range of square feet of building floor area that is the typical size for each building type.

- The Commercial Building Management Survey showed that $41 \%$ of commercial office buildings use some sort of EMS. In preparation of the tables, we have adjusted this by commercial building type and size to show that essentially $100 \%$ of some commercial building types use an EMS, and other types do not use them.

Some restaurant chains, like McDonald's, have done extensive energy efficiency studies and use EMSs in the restaurants, but typically, and for simplicity, we assume EMS is not used in food service buildings. Large retail stores such as Super Walmart or Costco that also sell groceries and have refrigeration equipment loads are in the category of grocery. Stores that sell primarily dry goods are in the category of retail.

Plug loads generally include coffee makers, copiers, computers, printers, ATMs, etc. In general, plug loads will ride through sags down to $75 \%$ of nominal for 2 seconds. Below this voltage, they drop out, and computer-based loads will go through a reboot sequence on voltage recovery.

The 3-minute column is really just for general interest. Three minutes is ancient history in planning for automatic response to power system transients. The 3-minute column estimates what the motor load would be doing at this point.

\section{Specific Motor Types and Applications}

The following is a discussion of specific equipment types given in the Appendix A tables, providing specific background and detail that may be of interest.

\section{- Roof Top Unit (RTU) Air Conditioner Compressor Motor}

RTU compressors motors are typically three-phase and include a dedicated local solid state control board. The motor contactors drop out at $58 \%$ to $61 \%$ voltage, which is higher than the stall voltage of about $50 \%$. Thus, it is unlikely that the three-phase motors will be stalling during transients, unlike the single-phase compressor motors. However, for unbalanced sags, stall may occur because the contactor voltage may stay near normal when the contactor is supplied by the voltages phases that are remaining near nominal voltage. SCE testing has shown that for both balanced and unbalanced voltages, after the contactor had dropped out due to low voltage, it would not reclose after voltage recovery for several minutes. Some RTUs may be equipped with manufacturer's low voltage protection that operates after 2 seconds at $80 \%$ voltage (SCE RTU test, page 36, 53).

\section{- Single-Phase Air-Conditioning Unit Compressor Motor}

Recent testing of single-phase (split-phase motor type) air conditioning units by SCE found that these units typically stall between $60 \%$ and $70 \%$ nominal voltage well before they are disconnected because their power contactors drop out at about 50\% to $60 \%$ voltage. It was also discovered that these single-phase compressor motors begin stalling rather quickly, normally within 3 cycles. This verifies the FIDVR theory that large numbers of singlephase air conditioning compressor motors may be stalling when voltage is between $70 \%$ and $50 \%$ of nominal. Singlephase compressor motors typically will not restart after tripping until a time delay of perhaps 3 minutes or more.

\section{- Local Solid State Control Board}

Reach-in and walk-in freezers, chillers, boiler elevators, and many other complex individual loads are also controlled by a local solid state control board. The board may be controlled by a local thermostat or by the EMS. 


\section{- Variable Frequency Drives}

Variable Frequency Drives (VFD) are typically programmed to ride through short duration voltage sags by current limiting the motor. In cases where only one phase is sagging, and the motor is being operated at partial load, the motor can run for several seconds or more. In the Appendix A tables, we assume that, for 60\% voltage and 5, 10, and 20 cycles, the VFD should be able to ride through by current limiting the motor. The VFD cannot ride through a 2-second or 3-minute loss of voltage unless it is equipped with energy storage. In testing, VFDs were noted to ride through sags of up to 2 seconds, or more, in duration, then trip after voltage recovery.

\section{- Chiller Motors}

Large chiller motors in the range of 100 to $700 \mathrm{HP}$ typically have their own proprietary local control board with voltage, overcurrent, and unbalance protection. Manufacturer's undervoltage protection is typically set at $90 \%$ of nominal voltage for 2 seconds and $80 \%$ of nominal voltage for 0.1 seconds. If the motor is de-energized on under voltage, until the built in anti-cycle timers minimum off delay expires.

\section{- Contactors}

Contactors, in general, will drop out within 5 cycles at $50 \%$ voltage. In some cases, voltage may sag to $40 \%$ before the contactor drops, and in some cases, it may be $60 \%$, but $50 \%$ is a good estimate. When the voltage recovers, at $70 \%$ of nominal voltage the BPA test shows the contactor reclosed after two cycles. At 65\%, it took 8.5 cycles to reclose. At $62 \%$ it never pulled in, even after multiple seconds.

The following graphic Fig. 1-1 represents reasonable estimates based on voltage sag and time; exact component trip points will be determined further by point on wave, motor load, control transformer load, ambient temperature. This graphic is for quick reference only, for more definitive parameters please see the Appendix A.

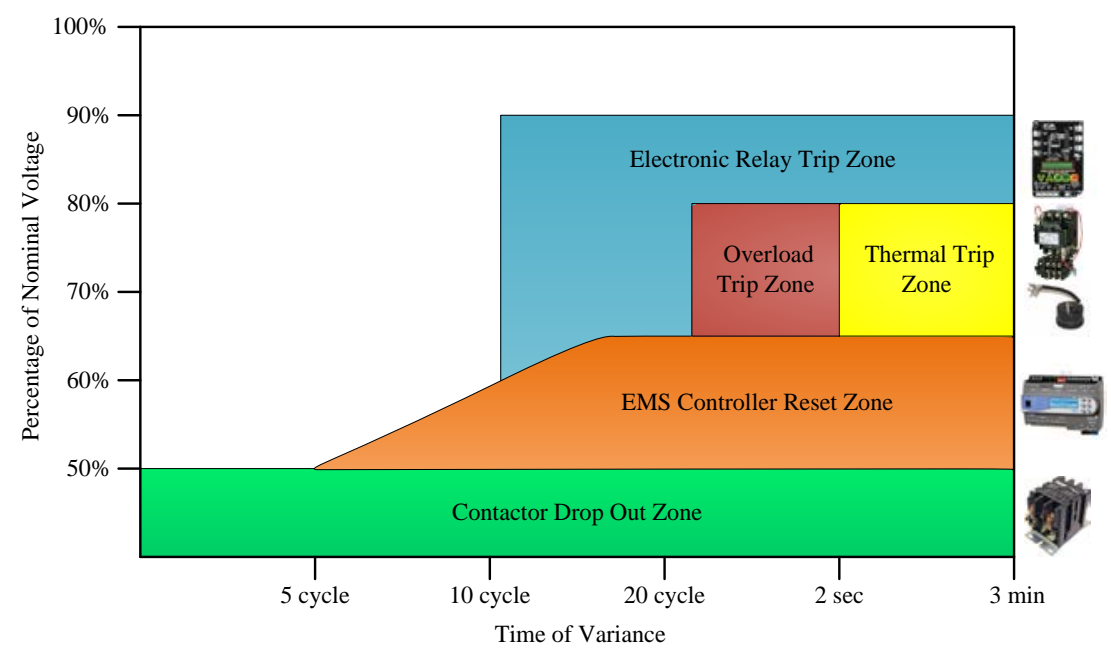

Fig. 1-1. Summary of specific type of motors

\section{- Energy Management System}

Testing at BPA has shown that the EMS can ride though severe voltage sags down to 65\% of nominal voltage, the EMS will drop out 2 to 3 seconds after the event and then takes 3 seconds to reset. This occurred even with a battery- 
backed EMS. If there are batteries in controllers that control the outputs to the equipment, it's typically just to keep the internal clock/calendar operating while power is not available. The internal batteries are not usually used to maintain normal operation during voltage dips or loss. It will be assumed for the tables in Appendix A that for voltage sag down to $65 \%$ of nominal, the EMS will ride through but not reset. For sags below 65\%, the EMS will ride through the initial event but will reset 2 seconds after the event, dropping the load until the programed sequences in the controller has run from the beginning. Some loads and motors will be started relatively quickly while others may take several minutes to reengage the loads. Sometimes the controllers have built in restart programs that delay startup of equipment. If an entire building were to lose power and then have it reapplied, having everything startup at once could cause issues. So the controllers are often set to delay at varying period so that restarting all the equipment won't cause any demand surcharges or other load issues. Testing revealed that the EMS controllers tripped less at voltages above $60 \%$ when their control transformer secondary was under $50 \%$ of its max VA capacity. EMS controllers did not trip on voltages above $65 \%$ when the loading of the transformer was at $80 \%$, which is the max allowable by the National Electrical Code. In general, testing showed that voltage variances below $60 \%$ resulted in the EMS controller resetting regardless of transformer loading. All EMS controllers that were tested showed no reaction to 5-cycle events even when the nominal voltage went to $0 \%$.

Fig. 1-2 reflects industry standard equipment start-up timing and sequencing in energy management systems. This does not reflect custom equipment start-up timing and sequencing for unique buildings or mechanical systems designated by a design engineer or equipment not controlled by an EMS. This graphic is for quick reference only; for more exact reset times please see the Appendix A.

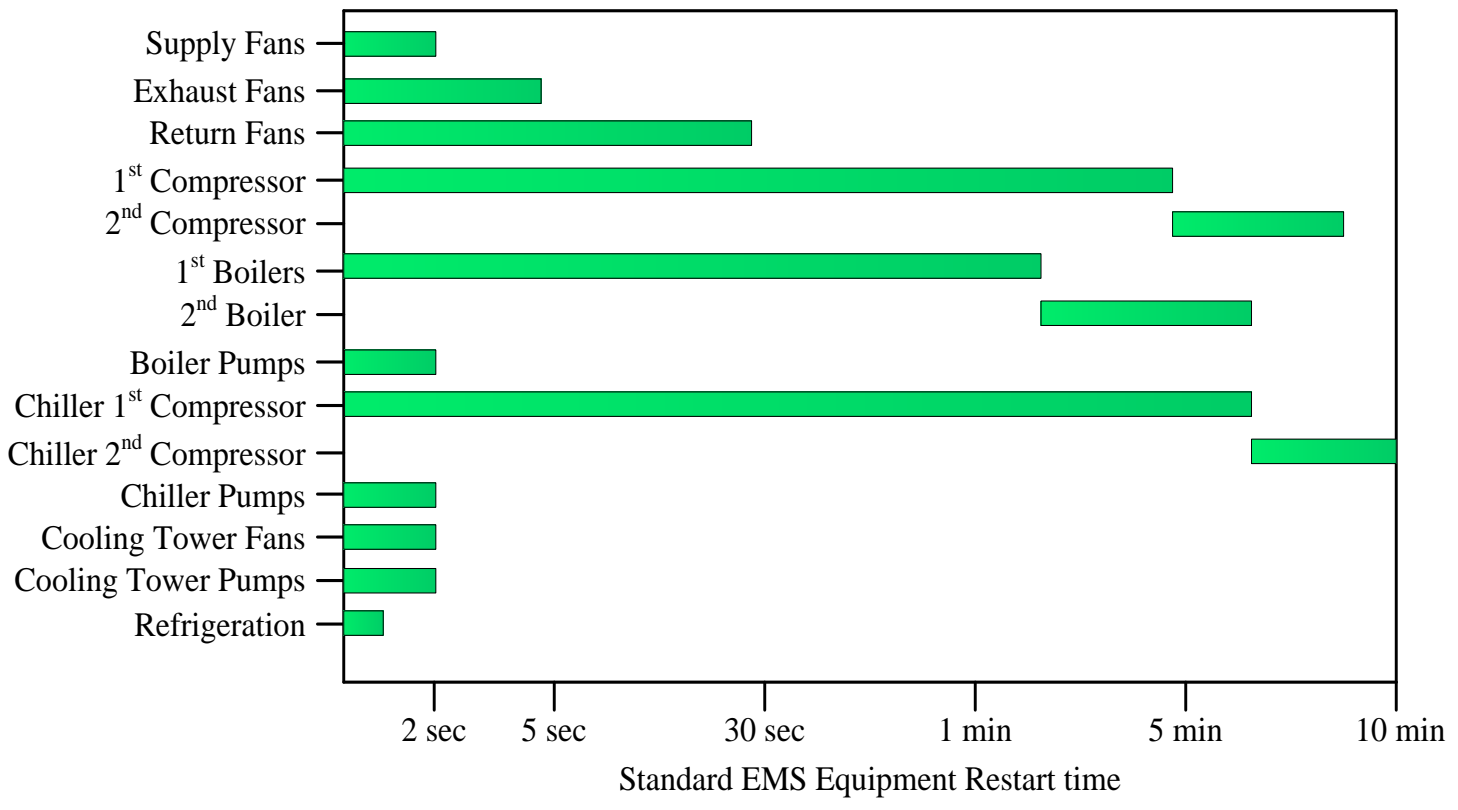

Fig. 1-2. Standard EMS equipment restart time 


\section{Appendix A: Building Motor Response Tables}

\section{A.1 Building Motor Response Tables for Voltage Sags down to $75 \%$ of Nominal Voltage}

Supermarket Building Load Table

Square feet: 50,000

Voltages above $75 \%$ of nominal

\begin{tabular}{|c|c|c|c|c|c|c|c|c|}
\hline Equipment & Motors & Protection & Controls & 5 cycle & 10 cycle & 20 cycle & 2 second & 3 minute \\
\hline $\begin{array}{l}\text { Refrigeration } \\
\text { and freezers }\end{array}$ & $\begin{array}{l}\text { 1-ph or 3-ph } \\
\text { compressor }\end{array}$ & $\begin{array}{l}\text { Fuse and } \\
\text { thermal }\end{array}$ & $\begin{array}{l}\text { Line voltage } \\
\text { with } \\
\text { contactor }\end{array}$ & \multicolumn{4}{|c|}{ Motor continues to run. } & $\begin{array}{l}\text { Extended sag } \\
\text { may have } \\
\text { caused the } \\
\text { thermal } \\
\text { protection to } \\
\text { trip. Auto } \\
\text { restart } \\
\text { after } \\
\text { manufacturer } \\
\text { solid state } \\
\text { controller } \\
\text { restart } \\
\text { sequence of } 3 \\
\text { to } 5 \text { min. }\end{array}$ \\
\hline \multirow[t]{3}{*}{$\begin{array}{l}\text { HVAC roof top } \\
\text { units (RTUs) }\end{array}$} & $\begin{array}{l}\text { 3-ph } \\
\text { compressor } \\
\text { motors }\end{array}$ & Fuse & \multirow{3}{*}{$\begin{array}{l}\text { Low voltage } \\
\text { thermostat } \\
\text { with } \\
\text { contactor }\end{array}$} & \multirow{3}{*}{\multicolumn{3}{|c|}{ Motors operate through voltage variance. }} & \multirow{3}{*}{\multicolumn{2}{|c|}{$\begin{array}{l}\text { Manufacturer control board may } \\
\text { have under voltage trip. } \\
\text { If tripped at } 2 \text { seconds, motor will } \\
\text { Auto restart. } \\
\text { Extended sag may have caused } \\
\text { the thermal protection to trip. }\end{array}$}} \\
\hline & $\begin{array}{l}\text { 3-ph indoor } \\
\text { fans motors }\end{array}$ & Fuse & & & & & & \\
\hline & $\begin{array}{l}\text { 1-ph } \\
\text { fractional } \\
\text { condenser and } \\
\text { induced draft } \\
\text { motors }\end{array}$ & $\begin{array}{l}\text { Fuse and } \\
\text { thermal }\end{array}$ & & & & & & \\
\hline $\begin{array}{l}\text { Misc plug } \\
\text { loads, } \\
\text { computers, } \\
\text { vending, } \\
\text { ATMs }\end{array}$ & $\begin{array}{l}\text { Exhaust fans } \\
\text { and } \\
\text { compressor } \\
\text { motors, single } \\
\text { phase, } \\
\text { fractional HP }\end{array}$ & $\begin{array}{l}\text { Thermal and } \\
\text { fuse }\end{array}$ & Local relay & \multicolumn{5}{|c|}{ Motors operate through voltage variance. } \\
\hline
\end{tabular}


Fast Food Building Load Table

Square feet: 2,500

Voltages above $75 \%$ of nominal

\begin{tabular}{|c|c|c|c|c|c|c|c|c|}
\hline Equipment & Motors & Protection & Controls & 5 cycle & 10 cycle & 20 cycle & 2 second & 3 minute \\
\hline \multirow[t]{2}{*}{$\begin{array}{l}\text { Walk-in } \\
\text { refrigeration } \\
\text { and freezers }\end{array}$} & $\begin{array}{l}\text { 1-ph or 3-ph } \\
\text { compressors }\end{array}$ & $\begin{array}{l}\text { Fuse and } \\
\text { thermal }\end{array}$ & $\begin{array}{l}\text { Line voltage } \\
\text { with contactor }\end{array}$ & \multicolumn{3}{|c|}{ Motors operate through voltage variance. } & \multicolumn{2}{|c|}{$\begin{array}{l}\text { Manufacturer control board may } \\
\text { have under voltage trip. } \\
\text { If tripped at } 2 \text { seconds, motor will } \\
\text { Auto restart. } \\
\text { Extended sag may have caused } \\
\text { the thermal protection to trip. }\end{array}$} \\
\hline & $\begin{array}{l}1 \text {-ph } \\
\text { fractional } \\
\text { evaporator } \\
\text { and } \\
\text { condenser } \\
\text { fan motors } \\
\end{array}$ & $\begin{array}{l}\text { Fuse and } \\
\text { thermal }\end{array}$ & $\begin{array}{l}\text { Line voltage } \\
\text { with contactor }\end{array}$ & \multicolumn{3}{|c|}{ Motors operate through voltage variance. } & \multicolumn{2}{|c|}{$\begin{array}{l}\text { Manufacturer control board may } \\
\text { have under voltage trip. } \\
\text { If tripped at } 2 \text { seconds, motor will } \\
\text { Auto restart. } \\
\text { Extended sag may have caused } \\
\text { the thermal protection to trip. }\end{array}$} \\
\hline \multirow[t]{3}{*}{ HVAC RTUs } & $\begin{array}{l}\text { 3-ph } \\
\text { compressors } \\
\text { motors }\end{array}$ & $\begin{array}{l}\text { Fuse and } \\
\text { thermal }\end{array}$ & $\begin{array}{l}\text { Low voltage } \\
\text { thermostat with } \\
\text { contactor }\end{array}$ & \multicolumn{3}{|c|}{ Motors operate through voltage variance. } & \multicolumn{2}{|c|}{$\begin{array}{l}\text { Manufacturer control board may } \\
\text { have under voltage trip. } \\
\text { If tripped at } 2 \text { seconds, motor will } \\
\text { Auto restart. } \\
\text { Extended sag may have caused } \\
\text { the thermal protection to trip. }\end{array}$} \\
\hline & $\begin{array}{l}\text { 3-ph } \\
\text { indoor fans } \\
\text { motors }\end{array}$ & Fuse & $\begin{array}{l}\text { Low Voltage } \\
\text { Thermostat } \\
\text { with contactor }\end{array}$ & \multicolumn{3}{|c|}{ Motors operate through voltage variance. } & \multicolumn{2}{|c|}{$\begin{array}{l}\text { Manufacturer control board may } \\
\text { have under voltage trip. } \\
\text { If tripped at } 2 \text { seconds, motor will } \\
\text { Auto restart }\end{array}$} \\
\hline & $\begin{array}{l}\text { 1-ph } \\
\text { fractional } \\
\text { condenser } \\
\text { and induced } \\
\text { draft motors }\end{array}$ & $\begin{array}{l}\text { Fuse and } \\
\text { thermal }\end{array}$ & $\begin{array}{l}\text { Low voltage } \\
\text { thermostat with } \\
\text { contactor }\end{array}$ & \multicolumn{3}{|c|}{ Motors operate through voltage variance. } & \multicolumn{2}{|c|}{$\begin{array}{l}\text { Manufacturer control board may } \\
\text { have under voltage trip. } \\
\text { If tripped at } 2 \text { seconds, motor will } \\
\text { Auto restart. } \\
\text { Extended sag may have caused } \\
\text { the thermal protection to trip. }\end{array}$} \\
\hline \multirow{2}{*}{$\begin{array}{l}\text { HVAC } \\
\text { make-up air } \\
\text { unit (MAU) } \\
\text { and exhaust } \\
\text { fan }\end{array}$} & $\begin{array}{l}\text { 3-ph motors } \\
\text { exhaust and } \\
\text { supply fan } \\
\text { motors } \\
\end{array}$ & $\begin{array}{l}\text { Fuse and } \\
\text { thermal }\end{array}$ & $\begin{array}{l}\text { Line voltage } \\
\text { start/stop } \\
\text { switch with } \\
\text { contactor } \\
\end{array}$ & \multicolumn{3}{|c|}{ Motors operate through voltage variance. } & \multicolumn{2}{|c|}{$\begin{array}{l}\text { Extended sag may have caused } \\
\text { the thermal protection to trip. }\end{array}$} \\
\hline & $\begin{array}{l}\text { 1-ph } \\
\text { fractional hp } \\
\text { induced } \\
\text { draft motors }\end{array}$ & $\begin{array}{l}\text { Fuse and } \\
\text { thermal }\end{array}$ & $\begin{array}{l}\text { Low voltage } \\
\text { thermostat with } \\
\text { contactor }\end{array}$ & \multicolumn{3}{|c|}{ Motors operate through voltage variance. } & \multicolumn{2}{|c|}{$\begin{array}{l}\text { Manufacturer control board may } \\
\text { have under voltage trip. } \\
\text { Extended sag may have caused } \\
\text { the thermal protection to trip. }\end{array}$} \\
\hline \multirow[t]{2}{*}{ Ice Machines } & $\begin{array}{l}\text { 1-ph motor } \\
\text { compressor }\end{array}$ & Thermal & $\begin{array}{l}\text { Solid-state } \\
\text { with contactor }\end{array}$ & \multicolumn{3}{|c|}{ Motors operate through voltage variance. } & \multicolumn{2}{|c|}{$\begin{array}{l}\text { Manufacturer control board may } \\
\text { have under voltage trip. Auto } \\
\text { restart after solid-state reboot and } \\
\text { program restart sequence. }\end{array}$} \\
\hline & $\begin{array}{l}\text { 1-ph } \\
\text { fractional } \\
\text { condenser } \\
\text { fan and } \\
\text { water pump } \\
\text { motors }\end{array}$ & Thermal & $\begin{array}{l}\text { Solid-state } \\
\text { with relay from } \\
\text { control board }\end{array}$ & \multicolumn{3}{|c|}{ Motors operate through voltage variance. } & \multicolumn{2}{|c|}{$\begin{array}{l}\text { Manufacturer control board may } \\
\text { have under voltage trip. Auto } \\
\text { restart after solid-state reboot and } \\
\text { program restart sequence. }\end{array}$} \\
\hline $\begin{array}{l}\text { Soft Serve } \\
\text { Ice Cream } \\
\text { Machine }\end{array}$ & $\begin{array}{l}\text { 1-ph or 3-ph } \\
\text { motor } \\
\text { compressor }\end{array}$ & Thermal & $\begin{array}{l}\text { Line voltage } \\
\text { with contactor }\end{array}$ & \multicolumn{3}{|c|}{ Motors operate through voltage variance. } & \multicolumn{2}{|c|}{$\begin{array}{l}\text { Manufacturer control board may } \\
\text { have under voltage trip. Auto } \\
\text { restart after solid-state reboot and } \\
\text { program restart sequence. }\end{array}$} \\
\hline
\end{tabular}




\begin{tabular}{|c|c|c|c|c|c|c|c|c|}
\hline Equipment & Motors & Protection & Controls & 5 cycle & 10 cycle & 20 cycle & 2 second & 3 minute \\
\hline & $\begin{array}{l}\text { 1-ph } \\
\text { fractional } \\
\text { condenser } \\
\text { fan and } \\
\text { pump } \\
\text { motors }\end{array}$ & Thermal & $\begin{array}{l}\text { Line voltage } \\
\text { with contactor }\end{array}$ & \multicolumn{3}{|c|}{ Motors operate through voltage variance. } & \multicolumn{2}{|c|}{$\begin{array}{l}\text { Manufacturer control board may } \\
\text { have under voltage trip. Auto } \\
\text { restart after solid-state reboot and } \\
\text { program restart sequence. }\end{array}$} \\
\hline $\begin{array}{l}\text { Reach-in } \\
\text { Refrigeration } \\
\text { and freezers }\end{array}$ & $\begin{array}{l}\text { 1-ph } \\
\text { fractional } \\
\text { compressor } \\
\text { condenser } \\
\text { and } \\
\text { evaporator } \\
\text { fan motors }\end{array}$ & Thermal & $\begin{array}{l}\text { Solid-state } \\
\text { with relay from } \\
\text { control board }\end{array}$ & \multicolumn{3}{|c|}{ Motors operate through voltage variance. } & \multicolumn{2}{|c|}{$\begin{array}{l}\text { Manufacturer control board may } \\
\text { have under voltage trip. Auto } \\
\text { restart after solid-state reboot and } \\
\text { program restart sequence. }\end{array}$} \\
\hline
\end{tabular}


Office Building Load Table

10,000 sf through $100,000 \mathrm{sf}$

Voltages above $75 \%$ of nominal

\begin{tabular}{|c|c|c|c|c|c|c|c|c|}
\hline Equipment & Motors & Protection & Controls & 5 cycle & 10 cycle & 20 cycle & 2 second & 3 minute \\
\hline \multirow[t]{2}{*}{$\begin{array}{l}\text { Custom } \\
\text { DX-AHUs } \\
\text { or AHUs } \\
\text { with air } \\
\text { cooled } \\
\text { chillers }\end{array}$} & $\begin{array}{l}\text { 3-ph } \\
\text { fan motors }\end{array}$ & $\begin{array}{l}\text { Over } \\
\text { voltage, } \\
\text { phase } \\
\text { imbalance, } \\
\text { over current, } \\
\text { current } \\
\text { limiting or } \\
\text { fuse and } \\
\text { thermal }\end{array}$ & $\begin{array}{l}\text { EMS with } \\
\text { VFD or } \\
\text { equipment } \\
\text { manufacturer } \\
\text { solid state } \\
\text { control tied } \\
\text { into ems }\end{array}$ & \multicolumn{4}{|c|}{$\begin{array}{l}\text { EMS remains in control. } \\
\text { Motors operate through voltage variance. }\end{array}$} & $\begin{array}{l}\text { Auto restart } \\
\text { after EMS } \\
\text { controller and } \\
\text { VFD reboot } \\
\text { and program } \\
\text { restart } \\
\text { sequence }\end{array}$ \\
\hline & $\begin{array}{l}\text { 3-ph } \\
\text { compressor } \\
\text { motors }\end{array}$ & $\begin{array}{l}\text { Electronic } \\
\text { Phase } \\
\text { Monitor } \\
\text { Fused And } \\
\text { Thermal }\end{array}$ & $\begin{array}{l}\text { EMS or } \\
\text { equipment } \\
\text { manufacturer } \\
\text { solid state } \\
\text { control tied } \\
\text { into EMS }\end{array}$ & \multicolumn{4}{|c|}{$\begin{array}{l}\text { EMS remains in control. } \\
\text { Motors operate through voltage variance. }\end{array}$} & $\begin{array}{l}\text { Manufacturer } \\
\text { control board } \\
\text { may have } \\
\text { under voltage } \\
\text { trip. Auto } \\
\text { restart } \\
\text { after controller } \\
\text { reboot and } \\
\text { program restart } \\
\text { sequence }\end{array}$ \\
\hline $\begin{array}{l}\text { Fan powered } \\
\text { VAVs }\end{array}$ & $\begin{array}{l}\text { 1-ph } \\
\text { fractional fan } \\
\text { motors }\end{array}$ & $\begin{array}{l}\text { Fuse and } \\
\text { Thermal }\end{array}$ & $\begin{array}{l}\text { EMS with } \\
\text { contactor }\end{array}$ & \multicolumn{4}{|c|}{$\begin{array}{l}\text { EMS remains in control. } \\
\text { Motors operate through voltage variance. }\end{array}$} & $\begin{array}{l}\text { Auto restart } \\
\text { after EMS } \\
\text { controller and } \\
\text { VFD reboot } \\
\text { and program } \\
\text { restart } \\
\text { sequence } \\
\end{array}$ \\
\hline $\begin{array}{l}\text { Fan coil } \\
\text { units }\end{array}$ & $\begin{array}{l}\text { 1-ph } \\
\text { Fractional } \\
\text { HP Fan } \\
\text { Motors }\end{array}$ & Thermal & $\begin{array}{l}\text { EMS with } \\
\text { contactor }\end{array}$ & \multicolumn{4}{|c|}{$\begin{array}{l}\text { EMS remains in control. } \\
\text { Motors operate through voltage variance. }\end{array}$} & $\begin{array}{l}\text { Auto restart } \\
\text { after EMS } \\
\text { controller and } \\
\text { VFD reboot } \\
\text { and program } \\
\text { restart } \\
\text { sequence }\end{array}$ \\
\hline $\begin{array}{l}\text { Building } \\
\text { general } \\
\text { exhaust fans }\end{array}$ & $\begin{array}{l}1 \text { and 3-ph } \\
\text { fan motors }\end{array}$ & $\begin{array}{l}\text { Over } \\
\text { voltage, } \\
\text { Phase } \\
\text { imbalance, } \\
\text { over current, } \\
\text { and current } \\
\text { limiting or } \\
\text { fuse and } \\
\text { thermal } \\
\end{array}$ & $\begin{array}{l}\text { EMS with } \\
\text { VFD or } \\
\text { line voltage } \\
\text { thermostats } \\
\text { or manual } \\
\text { start/stop } \\
\text { switch }\end{array}$ & \multicolumn{4}{|c|}{$\begin{array}{l}\text { EMS and VFD remains in control. } \\
\text { Motors operate through voltage variance. }\end{array}$} & $\begin{array}{l}\text { EMS controller } \\
\text { and VFD } \\
\text { reboot and } \\
\text { program restart } \\
\text { sequence / or } \\
\text { restart } \\
\text { instantaneously }\end{array}$ \\
\hline $\begin{array}{l}\text { CRAC } \\
\text { computer } \\
\text { room air } \\
\text { conditioner }\end{array}$ & $\begin{array}{l}\text { 1-ph or 3-ph } \\
\text { compressor }\end{array}$ & $\begin{array}{l}\text { Over } \\
\text { voltage, } \\
\text { phase } \\
\text { imbalance, } \\
\text { over current, } \\
\text { or fuse and } \\
\text { thermal }\end{array}$ & $\begin{array}{l}\text { Manufacturer } \\
\text { solid-state } \\
\text { with } \\
\text { contactor } \\
\text { or low } \\
\text { voltage } \\
\text { standalone } \\
\text { thermostats }\end{array}$ & \multicolumn{4}{|c|}{$\begin{array}{l}\text { Manufacturer solid-state remains in control. } \\
\text { Motors operate through voltage variance. }\end{array}$} & $\begin{array}{l}\text { Manufacturer } \\
\text { control board } \\
\text { may have } \\
\text { under voltage } \\
\text { trip. Auto } \\
\text { restart } \\
\text { after } \\
\text { manufacturer } \\
\text { solid state } \\
\text { controller } \\
\text { restart } \\
\text { sequence / or } \\
\text { restart after }\end{array}$ \\
\hline
\end{tabular}




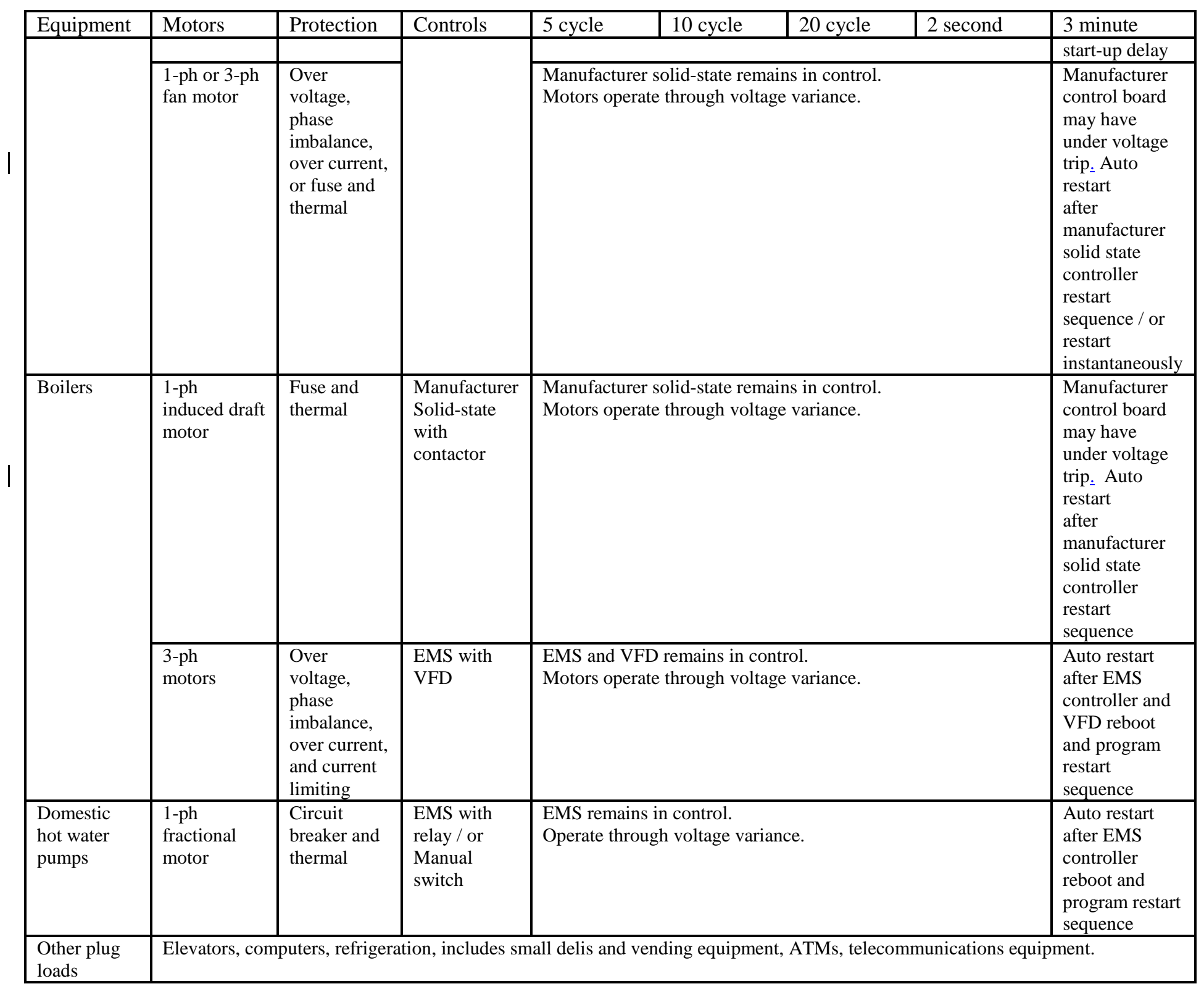


Office Building Load Table

Square feet: 100,000 to $1,000,000$

Voltages above $75 \%$ of nominal

\begin{tabular}{|c|c|c|c|c|c|c|c|c|}
\hline Equipment & Motors & Protection & Controls & 5 cycle & 10 cycle & 20 cycle & 2 second & 3 minute \\
\hline AHUs & $\begin{array}{l}\text { 3-ph fan } \\
\text { motors }\end{array}$ & $\begin{array}{l}\text { Over voltage, } \\
\text { phase } \\
\text { imbalance, } \\
\text { over current, } \\
\text { and current } \\
\text { limiting }\end{array}$ & $\begin{array}{l}\text { EMS with } \\
\text { VFD }\end{array}$ & \multicolumn{4}{|c|}{$\begin{array}{l}\text { EMS and VFD remains in control } \\
\text { Motors operate through voltage variance. }\end{array}$} & $\begin{array}{l}\text { Auto restart } \\
\text { after EMS } \\
\text { controller and } \\
\text { VFD reboot } \\
\text { and program } \\
\text { restart } \\
\text { sequence }\end{array}$ \\
\hline $\begin{array}{l}\text { Fan powered } \\
\text { VAVs }\end{array}$ & $\begin{array}{l}\text { 1-ph } \\
\text { fan motors }\end{array}$ & $\begin{array}{l}\text { Fuse and } \\
\text { thermal }\end{array}$ & $\begin{array}{l}\text { EMS with } \\
\text { contactor }\end{array}$ & \multicolumn{4}{|c|}{$\begin{array}{l}\text { EMS remains in control } \\
\text { Motors operate through voltage variance. }\end{array}$} & $\begin{array}{l}\text { Auto restart } \\
\text { after EMS } \\
\text { controller and } \\
\text { VFD reboot } \\
\text { and program } \\
\text { restart } \\
\text { sequence }\end{array}$ \\
\hline $\begin{array}{l}\text { (DOAS) } \\
\text { dedicated } \\
\text { outside air } \\
\text { system }\end{array}$ & $\begin{array}{l}\text { 3-ph } \\
\text { fan motors }\end{array}$ & $\begin{array}{l}\text { Over voltage, } \\
\text { phase } \\
\text { imbalance, } \\
\text { over current, } \\
\text { and current } \\
\text { limiting }\end{array}$ & $\begin{array}{l}\text { EMS with } \\
\text { VFD }\end{array}$ & \multicolumn{4}{|c|}{$\begin{array}{l}\text { EMS and VFD remains in control } \\
\text { Motors operate through voltage variance. }\end{array}$} & $\begin{array}{l}\begin{array}{l}\text { Auto restart } \\
\text { after EMS }\end{array} \\
\text { controller and } \\
\text { VFD reboot } \\
\text { and program } \\
\text { restart } \\
\text { sequence } \\
\end{array}$ \\
\hline \multirow[t]{2}{*}{ Chillers } & $\begin{array}{l}\text { 3-ph } \\
\text { compressor } \\
\text { motors }\end{array}$ & $\begin{array}{l}\text { Over voltage, } \\
\text { phase } \\
\text { imbalance, } \\
\text { over current }\end{array}$ & $\begin{array}{l}\text { Manufacturer } \\
\text { solid-state } \\
\text { controller tied } \\
\text { into EMS }\end{array}$ & \multicolumn{3}{|c|}{$\begin{array}{l}\text { Manufacturer Solid-state remains in control } \\
\text { Compressor operates through voltage } \\
\text { variance. }\end{array}$} & \multicolumn{2}{|c|}{$\begin{array}{l}\text { May trip offline due to under } \\
\text { voltage protection. Auto restart } \\
\text { after manufacturer solid state } \\
\text { controller restart sequence }\end{array}$} \\
\hline & $\begin{array}{l}\text { 3-ph } \\
\text { pump } \\
\text { motors }\end{array}$ & $\begin{array}{l}\text { Over voltage, } \\
\text { phase } \\
\text { imbalance, } \\
\text { over current, } \\
\text { and current } \\
\text { limiting }\end{array}$ & $\begin{array}{l}\text { EMS with } \\
\text { VFD }\end{array}$ & \multicolumn{4}{|c|}{$\begin{array}{l}\text { EMS and VFD remain in control. } \\
\text { Motors operate through voltage variance. }\end{array}$} & $\begin{array}{l}\text { Auto restart } \\
\text { after EMS } \\
\text { controller and } \\
\text { VFD reboot } \\
\text { and program } \\
\text { restart } \\
\text { sequence }\end{array}$ \\
\hline \multirow[t]{2}{*}{ Boilers } & $\begin{array}{l}\text { 1-ph } \\
\text { induced } \\
\text { draft motor }\end{array}$ & $\begin{array}{l}\text { Fuse and } \\
\text { Thermal }\end{array}$ & $\begin{array}{l}\text { Manufacturer } \\
\text { Solid-state } \\
\text { with contactor }\end{array}$ & \multicolumn{3}{|c|}{$\begin{array}{l}\text { EMS remains in control. } \\
\text { Motors operate through voltage variance. }\end{array}$} & $\begin{array}{l}\text { May trip offl } \\
\text { voltage prote } \\
\text { after manufa } \\
\text { controller re }\end{array}$ & $\begin{array}{l}\text { due to under } \\
\text { n. Auto restart } \\
\text { er solid state } \\
\text { t sequence }\end{array}$ \\
\hline & $\begin{array}{l}3 \text {-ph } \\
\text { motors }\end{array}$ & $\begin{array}{l}\text { Over voltage, } \\
\text { Phase } \\
\text { Imbalance, } \\
\text { over current, } \\
\text { and current } \\
\text { limiting }\end{array}$ & $\begin{array}{l}\text { EMS with } \\
\text { VFD }\end{array}$ & \multicolumn{3}{|c|}{$\begin{array}{l}\text { EMS remains in control. } \\
\text { Motors operate through voltage variance. }\end{array}$} & & 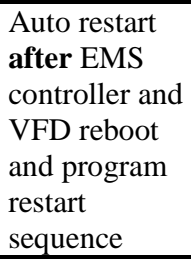 \\
\hline $\begin{array}{l}\text { Cooling } \\
\text { towers }\end{array}$ & $\begin{array}{l}\text { 3-ph } \\
\text { fan motor }\end{array}$ & $\begin{array}{l}\text { Over voltage, } \\
\text { Phase } \\
\text { Imbalance, } \\
\text { over current, } \\
\text { and current } \\
\text { limiting }\end{array}$ & $\begin{array}{l}\text { EMS with } \\
\text { VFD }\end{array}$ & \multicolumn{3}{|c|}{$\begin{array}{l}\text { EMS and VFD remain in control. } \\
\text { Motors operate through voltage variance. }\end{array}$} & & $\begin{array}{l}\text { Auto restart } \\
\text { after EMS } \\
\text { controller and } \\
\text { VFD reboot } \\
\text { and program } \\
\text { restart } \\
\text { sequence }\end{array}$ \\
\hline $\begin{array}{l}\text { Fan coil } \\
\text { units }\end{array}$ & $\begin{array}{l}1 \text {-ph } \\
\text { fractional } \\
\text { fan motors }\end{array}$ & $\begin{array}{l}\text { Fuse and } \\
\text { Thermal }\end{array}$ & $\begin{array}{l}\text { EMS with } \\
\text { Contactor }\end{array}$ & \multicolumn{3}{|c|}{$\begin{array}{l}\text { EMS remains in control. } \\
\text { Motors operate through voltage variance. }\end{array}$} & & $\begin{array}{l}\text { Auto restart } \\
\text { after EMS } \\
\text { controller and } \\
\text { VFD reboot } \\
\text { and program }\end{array}$ \\
\hline
\end{tabular}




\begin{tabular}{|c|c|c|c|c|c|c|c|c|}
\hline Equipment & Motors & Protection & Controls & 5 cycle & 10 cycle & 20 cycle & 2 second & 3 minute \\
\hline & & & & & & & & $\begin{array}{l}\text { restart } \\
\text { sequence }\end{array}$ \\
\hline $\begin{array}{l}\text { Building } \\
\text { general } \\
\text { exhaust fans }\end{array}$ & $\begin{array}{l}1 \text { and 3-ph } \\
\text { fan motors }\end{array}$ & $\begin{array}{l}\text { Over voltage, } \\
\text { Phase } \\
\text { Imbalance, } \\
\text { over current, } \\
\text { and current } \\
\text { limiting or } \\
\text { fuse and } \\
\text { thermal }\end{array}$ & $\begin{array}{l}\text { EMS with } \\
\text { VFD or } \\
\text { Line voltage } \\
\text { thermostat / } \\
\text { or manual } \\
\text { start/stop } \\
\text { switch }\end{array}$ & $\begin{array}{l}\text { EMS and V } \\
\text { Motors ope }\end{array}$ & $\begin{array}{l}\text { main in co } \\
\text { arough volt }\end{array}$ & riance. & & $\begin{array}{l}\text { Auto restart } \\
\text { after EMS } \\
\text { controller and } \\
\text { VFD reboot } \\
\text { and program } \\
\text { restart } \\
\text { sequence }\end{array}$ \\
\hline \multirow[t]{2}{*}{$\begin{array}{l}\text { (CRAC) } \\
\text { computer } \\
\text { room air } \\
\text { conditioner }\end{array}$} & $\begin{array}{l}\text { 1-ph or } \\
\text { 3-ph } \\
\text { compressor } \\
\text { motor }\end{array}$ & $\begin{array}{l}\text { Over voltage, } \\
\text { phase } \\
\text { imbalance, } \\
\text { over current, } \\
\text { or fuse and } \\
\text { thermal }\end{array}$ & \multirow[t]{2}{*}{$\begin{array}{l}\text { Manufacturer } \\
\text { solid-state } \\
\text { with contactor } \\
\text { / or low } \\
\text { voltage } \\
\text { standalone } \\
\text { thermostats }\end{array}$} & \multicolumn{3}{|c|}{$\begin{array}{l}\text { Manufacturer solid-state remains in control. } \\
\text { Compressors operate through voltage } \\
\text { variance. }\end{array}$} & \multicolumn{2}{|c|}{$\begin{array}{l}\text { May trip offline due to under } \\
\text { voltage protection. Auto restart } \\
\text { after manufacturer solid state } \\
\text { controller restart sequence }\end{array}$} \\
\hline & $\begin{array}{l}\text { 1-ph or 3- } \\
\text { ph } \\
\text { fan motor }\end{array}$ & $\begin{array}{l}\text { Over voltage, } \\
\text { phase } \\
\text { imbalance, } \\
\text { over current, } \\
\text { or fuse and } \\
\text { thermal }\end{array}$ & & \multicolumn{3}{|c|}{$\begin{array}{l}\text { Manufacturer solid-state remains in control } \\
\text { Motors operate through voltage variance. }\end{array}$} & \multicolumn{2}{|c|}{$\begin{array}{l}\text { May trip offline due to under } \\
\text { voltage protection. Auto restart } \\
\text { after manufacturer solid state } \\
\text { controller restart sequence }\end{array}$} \\
\hline $\begin{array}{l}\text { Domestic } \\
\text { cold water } \\
\text { pumps }\end{array}$ & $\begin{array}{l}\text { 3-ph } \\
\text { pump } \\
\text { motor }\end{array}$ & $\begin{array}{l}\text { Over voltage, } \\
\text { phase } \\
\text { imbalance, } \\
\text { over current, } \\
\text { and current } \\
\text { limiting }\end{array}$ & $\begin{array}{l}\text { Manufacturer } \\
\text { solid-state } \\
\text { with VFD }\end{array}$ & \multicolumn{3}{|c|}{$\begin{array}{l}\text { Manufacturer solid-state remains in control. } \\
\text { Motors operate through voltage variance. }\end{array}$} & $\begin{array}{l}\text { May trip offl } \\
\text { imbalance. A } \\
\text { equipment st }\end{array}$ & $\begin{array}{l}\text { on voltage } \\
\text { restart with } \\
\text { ig delay }\end{array}$ \\
\hline $\begin{array}{l}\text { Domestic hot } \\
\text { water pumps }\end{array}$ & $\begin{array}{l}\text { 1-ph } \\
\text { fractional } \\
\text { pump } \\
\text { motor }\end{array}$ & $\begin{array}{l}\text { Circuit } \\
\text { breaker and } \\
\text { thermal }\end{array}$ & $\begin{array}{l}\text { EMS with } \\
\text { relay / or } \\
\text { manual } \\
\text { switch }\end{array}$ & \multicolumn{3}{|c|}{$\begin{array}{l}\text { Manufacturer solid-state remains in control. } \\
\text { Motors operate through voltage variance. }\end{array}$} & & $\begin{array}{l}\text { May trip } \\
\text { offline due to } \\
\text { under voltage } \\
\text { protection. } \\
\text { Auto restart } \\
\text { after } \\
\text { manufacturer } \\
\text { solid state } \\
\text { controller } \\
\text { restart } \\
\text { sequence }\end{array}$ \\
\hline $\begin{array}{l}\text { Sewage } \\
\text { rejection }\end{array}$ & $\begin{array}{l}\text { 3-ph } \\
\text { pump } \\
\text { motor }\end{array}$ & $\begin{array}{l}\text { Fused with } \\
\text { current } \\
\text { overload }\end{array}$ & $\begin{array}{l}\text { Line voltage } \\
\text { mag-starter } \\
\text { start/stop } \\
\text { level switch }\end{array}$ & \multicolumn{5}{|c|}{ Motors operate through voltage variance. } \\
\hline Other loads & \multicolumn{8}{|c|}{$\begin{array}{l}\text { Elevators, computers, refrigeration, cooking includes small delis and vending equipment, ATMs, telecommunications equipment, } \\
\text { medical equipment, and manufacturing performed in commercial buildings. }\end{array}$} \\
\hline
\end{tabular}


Retail Building Load Table

Square feet: 5,000 and under

Voltage above $75 \%$

\begin{tabular}{|c|c|c|c|c|c|c|c|c|}
\hline Equipment & Motors & Protection & Controls & 5 cycle & 10 cycle & 20 cycle & 2 second & 3 minute \\
\hline \multirow[t]{4}{*}{$\begin{array}{l}\text { HVAC } \\
\text { RTUs }\end{array}$} & $\begin{array}{l}\text { 3-ph } \\
\text { compressor } \\
\text { motors }\end{array}$ & Fuse & \multirow{4}{*}{$\begin{array}{l}\text { Manufacturer } \\
\text { solid state } \\
\text { or } \\
\text { Low voltage } \\
\text { standalone } \\
\text { thermostats } \\
\text { manufacturer } \\
\text { solid state } \\
\text { or } \\
\text { Low voltage } \\
\text { standalone } \\
\text { thermostats }\end{array}$} & \multirow{4}{*}{\multicolumn{4}{|c|}{ Manufacturer control continues to operate. }} & \multirow{4}{*}{ 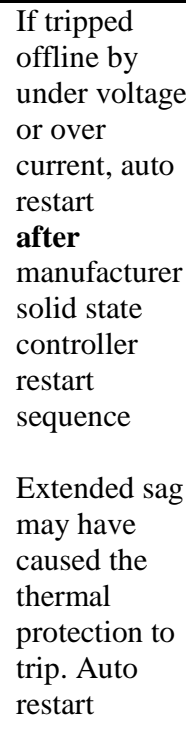 } \\
\hline & $\begin{array}{l}\text { 3-ph } \\
\text { indoor fans } \\
\text { motors }\end{array}$ & Fuse & & & & & & \\
\hline & $\begin{array}{l}\text { 1-ph } \\
\text { fractional } \\
\text { condenser } \\
\text { and induced } \\
\text { draft motors }\end{array}$ & $\begin{array}{l}\text { Fuse and } \\
\text { thermal }\end{array}$ & & & & & & \\
\hline & & & & & & & & \\
\hline Exhaust fans & $\begin{array}{l}1-\mathrm{ph} \\
\text { fractional } \\
\text { motor }\end{array}$ & $\begin{array}{l}\text { Circuit } \\
\text { breaker and } \\
\text { thermal }\end{array}$ & $\begin{array}{l}\text { Manual } \\
\text { switch }\end{array}$ & Motors op & ough volt & ance. & & $\begin{array}{l}\text { Extended sag } \\
\text { may have } \\
\text { caused the } \\
\text { thermal } \\
\text { protection to } \\
\text { trip. Auto } \\
\text { restart }\end{array}$ \\
\hline \multirow[t]{2}{*}{$\begin{array}{l}\text { Walk-in } \\
\text { refrigeration } \\
\text { and freezers }\end{array}$} & $\begin{array}{l}\text { 1-ph or 3-ph } \\
\text { compressors }\end{array}$ & $\begin{array}{l}\text { Fuse and } \\
\text { thermal }\end{array}$ & $\begin{array}{l}\text { Line voltage } \\
\text { with contactor }\end{array}$ & \multirow{2}{*}{\multicolumn{4}{|c|}{$\begin{array}{l}\text { Manufacturer control continues to operate. } \\
\text { Motors operate through voltage variance. }\end{array}$}} & $\begin{array}{l}\text { Extended sag } \\
\text { may have } \\
\text { caused the } \\
\text { thermal } \\
\text { protection to } \\
\text { trip. Auto } \\
\text { restart }\end{array}$ \\
\hline & $\begin{array}{l}\text { 1-ph } \\
\text { fractional } \\
\text { evaporator } \\
\text { and } \\
\text { condenser } \\
\text { fan motors }\end{array}$ & $\begin{array}{l}\text { Fuse and } \\
\text { thermal }\end{array}$ & $\begin{array}{l}\text { Line voltage } \\
\text { with contactor }\end{array}$ & & & & & $\begin{array}{l}\text { Extended sag } \\
\text { may have } \\
\text { caused the } \\
\text { thermal } \\
\text { protection to } \\
\text { trip. Auto } \\
\text { restart }\end{array}$ \\
\hline $\begin{array}{l}\text { Reach-in } \\
\text { refrigeration } \\
\text { and freezers }\end{array}$ & $\begin{array}{l}\text { 1-ph } \\
\text { fractional } \\
\text { compressor } \\
\text { motors }\end{array}$ & $\begin{array}{l}\text { Fuse and } \\
\text { thermal }\end{array}$ & $\begin{array}{l}\text { Manufacturer } \\
\text { solid-state } \\
\text { with contactor }\end{array}$ & \multicolumn{4}{|c|}{$\begin{array}{l}\text { Manufacturer solid-state remains in control. } \\
\text { Motors operate through voltage variance. }\end{array}$} & $\begin{array}{l}\text { If tripped by } \\
\text { thermal, auto } \\
\text { restart } \\
\text { after } \\
\text { manufacturer } \\
\text { solid state } \\
\text { controller } \\
\text { restart } \\
\text { sequence }\end{array}$ \\
\hline
\end{tabular}




\begin{tabular}{|c|c|c|c|c|c|c|c|c|}
\hline Equipment & Motors & Protection & Controls & 5 cycle & 10 cycle & 20 cycle & 2 second & 3 minute \\
\hline & $\begin{array}{l}\text { 1-ph } \\
\text { fractional } \\
\text { condenser } \\
\text { and } \\
\text { evaporator } \\
\text { fan motors }\end{array}$ & $\begin{array}{l}\text { Fuse and } \\
\text { Thermal }\end{array}$ & & \multicolumn{4}{|c|}{$\begin{array}{l}\text { Manufacturer solid-state remains in control. } \\
\text { Motors operate through voltage variance. }\end{array}$} & $\begin{array}{l}\text { If tripped } \\
\text { offline by } \\
\text { thermal, auto } \\
\text { restart } \\
\text { after } \\
\text { manufacturer } \\
\text { solid state } \\
\text { controller } \\
\text { restart } \\
\text { sequence }\end{array}$ \\
\hline $\begin{array}{l}\text { Other plug } \\
\text { loads }\end{array}$ & \multicolumn{8}{|c|}{ Gas pumps, refrigerated display cases, ice bins, TVs and monitors, cooking equipment } \\
\hline
\end{tabular}


Retail Building Load Table

Square feet: 15,000 to 40,000

Voltages above $75 \%$ of nominal

\begin{tabular}{|c|c|c|c|c|c|c|c|c|}
\hline Equipment & Motors & Protection & Controls & 5 cycle & 10 cycle & 20 cycle & 2 second & 3 minute \\
\hline \multirow[t]{4}{*}{$\begin{array}{l}\text { HVAC } \\
\text { RTUs }\end{array}$} & $\begin{array}{l}\text { 3-ph } \\
\text { compressor } \\
\text { motors }\end{array}$ & Fuse & \multirow{4}{*}{$\begin{array}{l}\text { EMS with } \\
\text { VFD } \\
\text { or } \\
\text { Manufacturer } \\
\text { solid state } \\
\text { tied into } \\
\text { EMS with } \\
\text { contactors } \\
\text { or } \\
\text { Low voltage } \\
\text { standalone } \\
\text { thermostats }\end{array}$} & \multirow{4}{*}{\multicolumn{4}{|c|}{$\begin{array}{l}\text { EMS and VFD remains in control. } \\
\text { Motors operate through voltage variance. }\end{array}$}} & \multirow{4}{*}{$\begin{array}{l}\text { If tripped } \\
\text { offline by under } \\
\text { voltage or } \\
\text { thermal } \\
\text { protection, auto } \\
\text { restart } \\
\text { after EMS } \\
\text { controller } \\
\text { reboot and } \\
\text { program restart } \\
\text { sequence / or } \\
\text { restart } \\
\text { instantaneously } \\
\text { Extended sag } \\
\text { may have } \\
\text { caused the } \\
\text { thermal } \\
\text { protection to } \\
\text { trip. Auto } \\
\text { restart }\end{array}$} \\
\hline & $\begin{array}{l}\text { 3-ph } \\
\text { indoor fans } \\
\text { motors }\end{array}$ & Fuse & & & & & & \\
\hline & $\begin{array}{l}\text { 1-ph } \\
\text { fractional } \\
\text { condenser } \\
\text { and induced } \\
\text { draft motors }\end{array}$ & $\begin{array}{l}\text { Fuse and } \\
\text { thermal }\end{array}$ & & & & & & \\
\hline & & & & & & & & \\
\hline $\begin{array}{l}\text { Exhaust } \\
\text { fans }\end{array}$ & $\begin{array}{l}\text { 1-ph } \\
\text { fractional } \\
\text { horsepower } \\
\text { motor } \\
\end{array}$ & $\begin{array}{l}\text { Circuit } \\
\text { breaker and } \\
\text { thermal }\end{array}$ & $\begin{array}{l}\text { EMS with } \\
\text { relay / or } \\
\text { manual } \\
\text { switch } \\
\end{array}$ & \multicolumn{5}{|c|}{$\begin{array}{l}\text { Motors operate through voltage variance } \\
\text { Extended sag may have caused the thermal protection to trip. Auto restart }\end{array}$} \\
\hline $\begin{array}{l}\text { Other plug } \\
\text { loads }\end{array}$ & \multicolumn{8}{|c|}{ Elevators, computers, refrigeration, cooking includes small delis and vending equipment, ATMs, telecommunications equipment } \\
\hline
\end{tabular}


Retail Building Load Table

Square feet: 15,000 to 100,000

Voltages above $75 \%$ of nominal

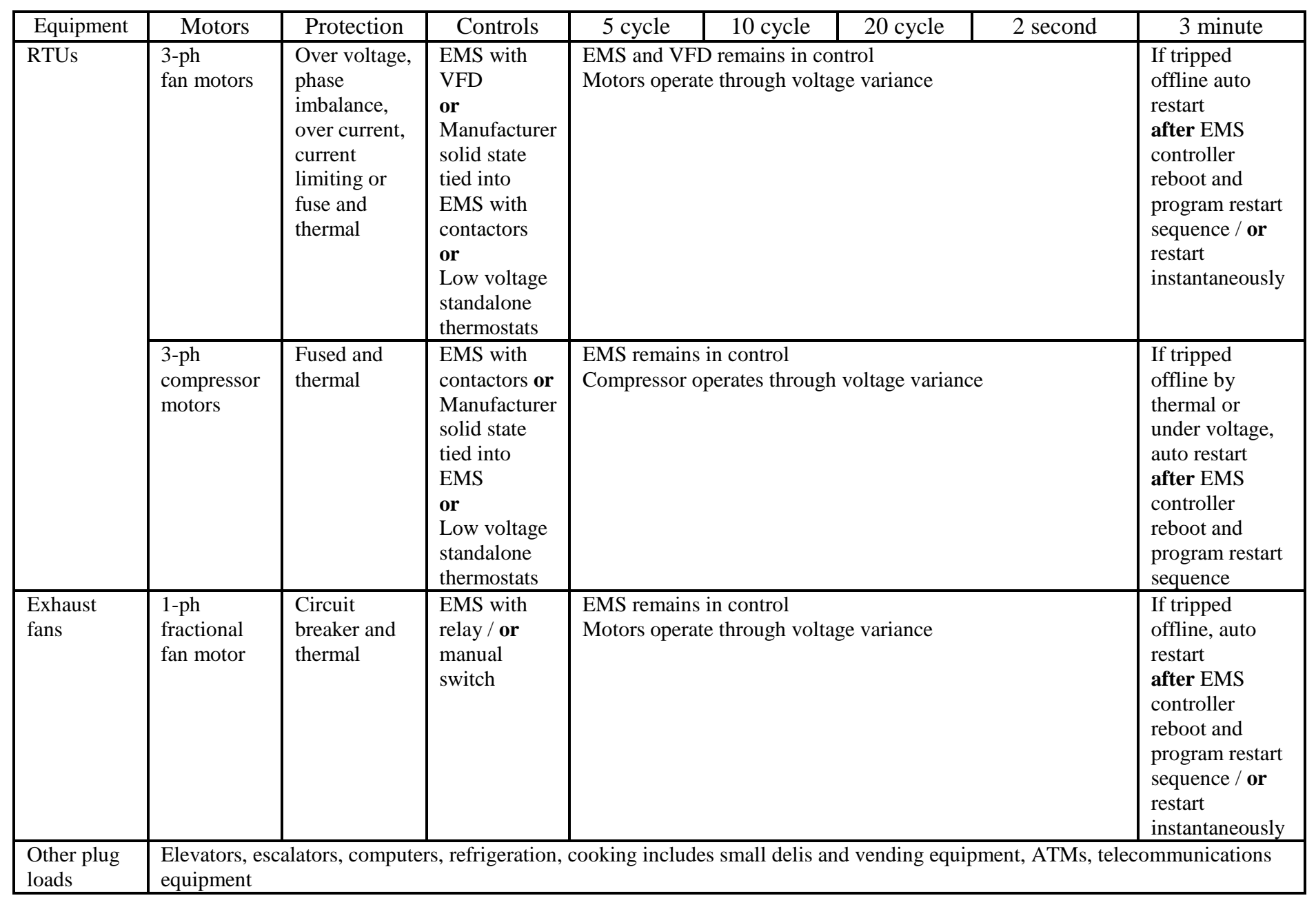


Warehouse Building Load Table

Square Feet 20,000

Voltages above $75 \%$ of nominal

\begin{tabular}{|c|c|c|c|c|c|c|c|c|}
\hline Equipment & Motors & Protection & Controls & 5 cycle & 10 cycle & 20 cycle & 2 second & 3 minute \\
\hline $\begin{array}{l}\text { Gas fired unit } \\
\text { heaters }\end{array}$ & $\begin{array}{l}\text { 1-ph } \\
\text { fractional fan } \\
\text { motors }\end{array}$ & Thermal & $\begin{array}{l}\text { Low voltage } \\
\text { thermostat } \\
\text { switch with } \\
\text { contactor }\end{array}$ & \multicolumn{4}{|c|}{ Motors operate through voltage variance } & $\begin{array}{l}\text { Thermostat } \\
\text { reboot and } \\
\text { program } \\
\text { restart } \\
\text { sequence }\end{array}$ \\
\hline Exhaust fans & $\begin{array}{l}\text { 1-ph } \\
\text { fan motors }\end{array}$ & Thermal & $\begin{array}{l}\text { Line voltage } \\
\text { start/stop } \\
\text { switch with } \\
\text { contactor }\end{array}$ & \multicolumn{5}{|c|}{$\begin{array}{l}\text { Motors operate through voltage variance } \\
\text { Extended sag may have caused the thermal protection to trip. Auto restart }\end{array}$} \\
\hline Paddle fans & $\begin{array}{l}\text { 1-ph } \\
\text { fan motors }\end{array}$ & Thermal & $\begin{array}{l}\text { Manual } \\
\text { start/stop } \\
\text { switch }\end{array}$ & \multicolumn{5}{|c|}{$\begin{array}{l}\text { Motors operate through voltage variance } \\
\text { Extended sag may have caused the thermal protection to trip. Auto restart }\end{array}$} \\
\hline $\begin{array}{l}\text { Other plug } \\
\text { loads }\end{array}$ & & & & & & & $s, b$ & \\
\hline
\end{tabular}




\title{
A.2 Building Motor Response Tables for Voltage Variance Range of 75 to $\mathbf{5 0 \%}$ of Nominal
}

\author{
Supermarket Building Load Table
}

Square feet: 50,000 (Albertson's)

Voltages between $75 \%$ and $50 \%$ of nominal

\begin{tabular}{|c|c|c|c|c|c|c|c|c|}
\hline Equipment & Motors & Protection & Controls & 5 cycle & 10 cycle & 20 cycle & 2 second & 3 minute \\
\hline \multirow[t]{3}{*}{ RTUs } & $\begin{array}{l}\text { 3-ph indoor } \\
\text { fan motors }\end{array}$ & $\begin{array}{l}\text { Fused } \\
\text { possibly } \\
\text { voltage } \\
\text { monitored } \\
\text { possibly } \\
\text { overload } \\
\text { relays }\end{array}$ & $\begin{array}{l}\text { EMS with } \\
\text { contactors } \\
\text { or } \\
\text { Manufacturer } \\
\text { solid state } \\
\text { tied into } \\
\text { EMS with } \\
\text { contactors } \\
\text { or } \\
\text { Low voltage } \\
\text { standalone } \\
\text { thermostats }\end{array}$ & \multirow[t]{3}{*}{$\begin{array}{l}\text { EMS } \\
\text { remains in } \\
\text { control } \\
\text { Contactor } \\
\text { drops out at } \\
50 \% \text { V and } \\
\text { reenergizes } \\
\text { after } 1 \text { to } 8 \\
\text { cycles after } \\
\text { event }\end{array}$} & \multicolumn{4}{|c|}{$\begin{array}{l}\text { EMS and VFD drop out } 2 \text { seconds after event below } 65 \% \mathrm{~V} \text {, but } \\
\text { will automatically restart with voltage recovery. First fan starts } \\
\text { within } 5 \text { seconds, second fan if applicable restarts at } 30 \text { seconds. } \\
\text { Contactors operate through voltage variance, but drop out when } \\
\text { EMS drops offline } 2 \text { seconds after event. Or, if the voltage dips } \\
\text { below } 50 \% \mathrm{~V} \text {, the contactor will drop and reenergize } 1 \text { to } 8 \text { cycles } \\
\text { after event, and then drop out again when the EMS drops } 2 \\
\text { seconds later. } \\
\text { Possible overload trip if the voltage variance is longer than } 4 \text { to } 5 \\
\text { seconds and above } 65 \% \mathrm{~V} \text {. }\end{array}$} \\
\hline & $\begin{array}{l}\text { 3-ph } \\
\text { compressor } \\
\text { motors }\end{array}$ & $\begin{array}{l}\text { Fused and } \\
\text { thermal } \\
\text { possibly } \\
\text { voltage } \\
\text { monitored }\end{array}$ & $\begin{array}{l}\text { EMS with } \\
\text { contactors } \\
\text { or } \\
\text { Manufacturer } \\
\text { solid state } \\
\text { tied into } \\
\text { EMS } \\
\text { or } \\
\text { Low voltage } \\
\text { standalone } \\
\text { thermostats }\end{array}$ & & \multicolumn{4}{|c|}{$\begin{array}{l}\text { EMS drops out } 2 \text { seconds after event below } 65 \% \mathrm{~V} \text {, but will } \\
\text { automatically restart with voltage recovery; } 270 \text { seconds first } \\
\text { compressor, } 150 \text {-second interstaging delay for each additional } \\
\text { compressor if applicable. } \\
\text { Contactors operate through voltage variance but drop out when } \\
\text { EMS drops offline } 2 \text { seconds after event. Or, if the voltage dips } \\
\text { below } 50 \% \mathrm{~V} \text {, the contactor will drop and reenergize } 1 \text { to } 8 \text { cycles } \\
\text { after event, and then drop out again when the EMS drops } 2 \\
\text { seconds later. } \\
\text { Possible thermal trip if the voltage variance is longer than } 4 \text { to } 5 \\
\text { seconds and above } 65 \% \mathrm{~V} \text {. }\end{array}$} \\
\hline & $\begin{array}{l}\text { 1-ph } \\
\text { fractional } \\
\text { condenser } \\
\text { and induced } \\
\text { draft motors }\end{array}$ & $\begin{array}{l}\text { Fused and } \\
\text { thermal } \\
\text { possibly } \\
\text { voltage } \\
\text { monitored }\end{array}$ & $\begin{array}{l}\text { EMS with } \\
\text { contactors or } \\
\text { Manufacturer } \\
\text { solid state } \\
\text { tied into } \\
\text { EMS } \\
\text { or } \\
\text { Low voltage } \\
\text { standalone } \\
\text { thermostats }\end{array}$ & & \multicolumn{4}{|c|}{$\begin{array}{l}\text { EMS and VFD drop out } 2 \text { seconds after event below } 65 \% \mathrm{~V} \text {, but } \\
\text { will automatically restart. } 270 \text { seconds with the first compressor, } \\
150 \text {-second interstaging delay for each additional compressor if } \\
\text { applicable. } \\
\text { Contactors operate through voltage variance but drop out when } \\
\text { EMS drops offline } 2 \text { seconds after event. Or, if the voltage dips } \\
\text { below } 50 \% \mathrm{~V} \text {, the contactor will drop and reenergize } 1 \text { to } 8 \text { cycles } \\
\text { after event, and then drop out again when the EMS drops } 2 \\
\text { seconds later. } \\
\text { Possible thermal trip if the voltage variance is longer than } 4 \text { to } 5 \\
\text { seconds and above } 65 \% \mathrm{~V} \text {. }\end{array}$} \\
\hline $\begin{array}{l}\text { Refrigeration } \\
\text { and freezers }\end{array}$ & $\begin{array}{l}\text { 1-ph or } \\
\text { 3-ph } \\
\text { compressor } \\
\text { motors }\end{array}$ & $\begin{array}{l}\text { Fuse and } \\
\text { Thermal }\end{array}$ & $\begin{array}{l}\text { Line voltage } \\
\text { with } \\
\text { contactor }\end{array}$ & $\begin{array}{l}\text { Contactor } \\
\text { drops out at } \\
50 \% \mathrm{~V} \text { and } \\
\text { reenergizes } \\
\text { after } 1 \text { to } 8 \\
\text { cycles after } \\
\text { event }\end{array}$ & \multicolumn{4}{|c|}{$\begin{array}{l}\text { Automatically restart. } 300 \text { seconds for compressor and all } \\
\text { associated 1-ph fans. }\end{array}$} \\
\hline $\begin{array}{l}\text { Misc plug } \\
\text { loads }\end{array}$ & \multicolumn{8}{|c|}{$\begin{array}{l}\text { Exhaust fans and compressor motors, single phase, fractional HP drop out at } 50 \% \text { to } 60 \% \text { voltage, time delay on restart of } \\
\text { computer-based loads. }\end{array}$} \\
\hline
\end{tabular}


Fast Food Building Load Table

Square feet: 2,500

Voltages between $75 \%$ and $50 \%$ of nominal

\begin{tabular}{|c|c|c|c|c|c|c|c|c|}
\hline Equipment & Motors & Protection & Controls & 5 cycle & 10 cycle & 20 cycle & 2 second & 3 minute \\
\hline \multirow{2}{*}{$\begin{array}{l}\text { Walk-in } \\
\text { refrigeration } \\
\text { and freezers }\end{array}$} & $\begin{array}{l}\text { 1-ph or 3-ph } \\
\text { compressors }\end{array}$ & $\begin{array}{l}\text { Fuse and } \\
\text { thermal }\end{array}$ & $\begin{array}{l}\text { Line voltage } \\
\text { with contactor }\end{array}$ & \multirow{2}{*}{$\begin{array}{l}\text { Contactor drops } \\
\text { out at } 50 \% \mathrm{~V} \text {. } \\
\text { Reenergizes after } 1 \\
\text { to } 8 \text { cycles after } \\
\text { event. }\end{array}$} & \multirow{2}{*}{\multicolumn{4}{|c|}{$\begin{array}{l}\text { Automatically restart. } 300 \text { seconds for compressor and } \\
\text { all associated 1-phase fans. } \\
\text { Possible thermal trip if the voltage variance is longer } \\
\text { than } 2 \text { to } 3 \text { seconds and above } 50 \% \mathrm{~V} \text {. }\end{array}$}} \\
\hline & $\begin{array}{l}\text { 1-ph } \\
\text { fractional } \\
\text { evaporator } \\
\text { and } \\
\text { condenser } \\
\text { fan motors }\end{array}$ & $\begin{array}{l}\text { Fuse and } \\
\text { thermal }\end{array}$ & $\begin{array}{l}\text { Line voltage } \\
\text { with contactor }\end{array}$ & & & & & \\
\hline \multirow[t]{3}{*}{ RTUs } & $\begin{array}{l}\text { 3-ph indoor } \\
\text { fan motors }\end{array}$ & $\begin{array}{l}\text { Fused } \\
\text { Possibly } \\
\text { Overload } \\
\text { relays }\end{array}$ & $\begin{array}{l}\text { Low voltage } \\
\text { thermostat with } \\
\text { contactor }\end{array}$ & \multirow{3}{*}{$\begin{array}{l}\text { Thermostat } \\
\text { remains in control. } \\
\text { Contactor drops } \\
\text { out at } 50 \% \mathrm{~V} \text { and } \\
\text { reenergizes after } 1 \\
\text { to } 8 \text { cycles after } \\
\text { event. }\end{array}$} & \multicolumn{4}{|c|}{$\begin{array}{l}\text { Thermostat will drop out below } 65 \% \mathrm{~V} \text {, but will } \\
\text { automatically restart. Fan starts within } 30 \text { seconds. } \\
\text { Possible overload trip if the voltage variance is long } \\
\text { enough and above } 65 \% \text {. }\end{array}$} \\
\hline & $\begin{array}{l}\text { 3-ph } \\
\text { compressor } \\
\text { motors }\end{array}$ & $\begin{array}{l}\text { Fuse and } \\
\text { thermal }\end{array}$ & $\begin{array}{l}\text { Low voltage } \\
\text { thermostat with } \\
\text { contactor }\end{array}$ & & \multicolumn{4}{|c|}{$\begin{array}{l}\text { Thermostat will drop out below } 65 \% \mathrm{~V} \text {, but will } \\
\text { automatically restart the compressor within } 300 \text { seconds. } \\
\text { Possible thermal trip if the voltage variance is longer } \\
\text { than } 4 \text { to } 5 \text { seconds and above } 65 \% \mathrm{~V} \text {. }\end{array}$} \\
\hline & $\begin{array}{l}\text { 1-ph } \\
\text { fractional } \\
\text { condenser } \\
\text { and induced } \\
\text { draft motors }\end{array}$ & $\begin{array}{l}\text { Fuse and } \\
\text { thermal }\end{array}$ & $\begin{array}{l}\text { Low voltage } \\
\text { thermostat with } \\
\text { contactor }\end{array}$ & & \multicolumn{4}{|c|}{$\begin{array}{l}\text { Thermostat will drop out below } 65 \% \mathrm{~V} \text {, but will } \\
\text { automatically restart the fans with the compressor within } \\
300 \text { seconds. } \\
\text { Possible thermal trip if the voltage variance is longer } \\
\text { than } 4 \text { to } 5 \text { seconds and above } 65 \% \mathrm{~V} \text {. }\end{array}$} \\
\hline \multirow{2}{*}{$\begin{array}{l}\text { HVAC } \\
\text { make-up air } \\
\text { unit MAU } \\
\text { and exhaust } \\
\text { fan }\end{array}$} & $\begin{array}{l}\text { 3-ph motors } \\
\text { exhaust and } \\
\text { supply fan } \\
\text { motors }\end{array}$ & $\begin{array}{l}\text { Fused } \\
\text { possibly } \\
\text { overload } \\
\text { relays } \\
\end{array}$ & $\begin{array}{l}\text { Line voltage } \\
\text { start/stop } \\
\text { switch with } \\
\text { contactor }\end{array}$ & \multicolumn{5}{|c|}{$\begin{array}{l}\text { Contactor drops out at } 50 \% \mathrm{~V} \text {. } \\
\text { Reenergizes after } 1 \text { to } 8 \text { cycles after event. } \\
\text { Possible overload trip if the voltage variance is long enough and above } 50 \% \text {. }\end{array}$} \\
\hline & $\begin{array}{l}\text { 1-ph } \\
\text { fractional } \\
\text { HP induced } \\
\text { draft motors }\end{array}$ & $\begin{array}{l}\text { Fuse and } \\
\text { thermal }\end{array}$ & $\begin{array}{l}\text { Low voltage } \\
\text { thermostat with } \\
\text { contactor }\end{array}$ & $\begin{array}{l}\text { Thermostat } \\
\text { remains in control. } \\
\text { Contactor drops } \\
\text { out at } 50 \% \mathrm{~V} \text { and } \\
\text { reenergizes after } 1 \\
\text { to } 8 \text { cycles after } \\
\text { event. }\end{array}$ & \multicolumn{4}{|c|}{$\begin{array}{l}\text { Thermostat will drop out below } 65 \% \mathrm{~V} \text {, but will } \\
\text { automatically restart the Induced draft motors. } \\
\text { Possible thermal trip if the voltage variance is longer } \\
\text { than } 4 \text { to } 5 \text { seconds and above } 65 \% \mathrm{~V}\end{array}$} \\
\hline \multirow[t]{2}{*}{ Ice machines } & $\begin{array}{l}\text { 1-ph motor } \\
\text { compressor }\end{array}$ & Thermal & $\begin{array}{l}\text { Solid-state } \\
\text { with contactor }\end{array}$ & $\begin{array}{l}\text { Control board } \\
\text { remains in control. } \\
\text { Contactor drops } \\
\text { out at } 50 \% \mathrm{~V} \text { and } \\
\text { reenergizes after } 1 \\
\text { to } 8 \text { cycles after } \\
\text { event. }\end{array}$ & \multicolumn{4}{|c|}{$\begin{array}{l}\text { Solid-state control board will drop out below } 65 \% \mathrm{~V} \text {. } \\
\text { Manual reset needed. } \\
\text { Possible thermal trip if the voltage variance is longer } \\
\text { than } 4 \text { to } 5 \text { seconds and above } 65 \% \mathrm{~V} \text {. }\end{array}$} \\
\hline & $\begin{array}{l}\text { 1-ph } \\
\text { fractional } \\
\text { condenser } \\
\text { fan and } \\
\text { water pump } \\
\text { motors } \\
\end{array}$ & Thermal & $\begin{array}{l}\text { Solid-state } \\
\text { with relay from } \\
\text { control board }\end{array}$ & $\begin{array}{l}\text { Motor will } \\
\text { continue to operate } \\
\text { through voltage } \\
\text { variance. }\end{array}$ & \multicolumn{4}{|c|}{$\begin{array}{l}\text { Solid-state control board will drop out below } 65 \% \mathrm{~V} \text {. } \\
\text { Manual reset needed. } \\
\text { Possible thermal trip if the voltage variance is longer } \\
\text { than } 4 \text { to } 5 \text { seconds and above } 65 \% \mathrm{~V} \text {. }\end{array}$} \\
\hline
\end{tabular}




\begin{tabular}{|c|c|c|c|c|c|c|c|c|}
\hline Equipment & Motors & Protection & Controls & 5 cycle & 10 cycle & 20 cycle & 2 second & 3 minute \\
\hline \multirow[t]{2}{*}{$\begin{array}{l}\text { Soft serve } \\
\text { ice cream } \\
\text { machine }\end{array}$} & $\begin{array}{l}\text { 1-ph motor } \\
\text { compressor }\end{array}$ & Thermal & $\begin{array}{l}\text { Solid-state } \\
\text { with contactor }\end{array}$ & \multirow{2}{*}{$\begin{array}{l}\text { Control board } \\
\text { remains in control. } \\
\text { Contactor drops } \\
\text { out at } 50 \% \mathrm{~V} \text { and } \\
\text { reenergizes after } 1 \\
\text { to } 8 \text { cycles after } \\
\text { event. }\end{array}$} & \multicolumn{4}{|c|}{$\begin{array}{l}\text { Solid-state control board will drop out below } 65 \% \mathrm{~V} \text {. } \\
\text { Manual reset needed. } \\
\text { Possible thermal trip if the voltage variance is longer } \\
\text { than } 4 \text { to } 5 \text { seconds and above } 65 \% \mathrm{~V} \text {. }\end{array}$} \\
\hline & $\begin{array}{l}\text { 1-ph } \\
\text { fractional } \\
\text { condenser } \\
\text { fan and } \\
\text { water pump } \\
\text { motors }\end{array}$ & Thermal & $\begin{array}{l}\text { Solid-state } \\
\text { with contactor }\end{array}$ & & \multicolumn{4}{|c|}{$\begin{array}{l}\text { Solid-state control board will drop out below } 65 \% \mathrm{~V} \text {. } \\
\text { Manual reset needed. } \\
\text { Possible thermal trip if the voltage variance is longer } \\
\text { than } 4 \text { to } 5 \text { seconds and above } 65 \% \mathrm{~V} \text {. }\end{array}$} \\
\hline $\begin{array}{l}\text { Reach-in } \\
\text { refrigeration } \\
\text { and freezers }\end{array}$ & $\begin{array}{l}\text { 1-ph } \\
\text { fractional } \\
\text { compressor } \\
\text { condenser } \\
\text { and } \\
\text { evaporator } \\
\text { fan motors }\end{array}$ & Thermal & $\begin{array}{l}\text { Solid-state } \\
\text { with relay from } \\
\text { control board }\end{array}$ & $\begin{array}{l}\text { Motor will } \\
\text { continue to operate } \\
\text { through voltage } \\
\text { variance. }\end{array}$ & \multicolumn{4}{|c|}{$\begin{array}{l}\text { Solid-state control board will drop out below } 65 \% \mathrm{~V} \text {. } \\
\text { Manual reset needed. } \\
\text { Possible thermal trip if the voltage variance is longer } \\
\text { than } 4 \text { to } 5 \text { seconds and above } 65 \% \mathrm{~V} \text {. }\end{array}$} \\
\hline
\end{tabular}


Office Building Load Table

Square feet: 10,000 through 100,000

Voltages between $75 \%$ and $50 \%$ of nominal

\begin{tabular}{|c|c|c|c|c|c|c|c|c|}
\hline Equipment & Motors & Protection & Controls & 5 cycle & 10 cycle & 20 cycle & 2 second & 3 minute \\
\hline \multirow[t]{2}{*}{$\begin{array}{l}\text { Custom } \\
\text { DX-AHUs } \\
\text { or AHUs } \\
\text { with air } \\
\text { cooled } \\
\text { chillers }\end{array}$} & $\begin{array}{l}\text { 3-ph } \\
\text { fan motors }\end{array}$ & $\begin{array}{l}\text { Over voltage, } \\
\text { phase } \\
\text { imbalance, } \\
\text { over current, } \\
\text { or fuse and } \\
\text { thermal }\end{array}$ & $\begin{array}{l}\text { EMS with } \\
\text { VFD or } \\
\text { equipment } \\
\text { manu- } \\
\text { facturer } \\
\text { solid state } \\
\text { control tied } \\
\text { into EMS }\end{array}$ & $\begin{array}{l}\text { EMS } \\
\text { remains in } \\
\text { control. }\end{array}$ & \multicolumn{4}{|c|}{$\begin{array}{l}\text { EMS and VFD operate through event then drop out } 2 \text { seconds after } \\
\text { event below } 65 \% \text { V, but will automatically restart. First fan starts } \\
\text { within } 5 \text { seconds, second fan if applicable restarts at } 30 \text { seconds. }\end{array}$} \\
\hline & $\begin{array}{l}\text { 3-ph } \\
\text { compressor } \\
\text { motors }\end{array}$ & $\begin{array}{l}\text { Electronic } \\
\text { phase } \\
\text { monitor } \\
\text { fused and } \\
\text { thermal }\end{array}$ & $\begin{array}{l}\text { EMS or } \\
\text { equipment } \\
\text { manufactur } \\
\text { ers solid } \\
\text { state } \\
\text { control tied } \\
\text { into EMS }\end{array}$ & $\begin{array}{l}\text { EMS } \\
\text { remains in } \\
\text { control. } \\
\text { Contactor } \\
\text { drops out at } \\
50 \% \mathrm{~V} \text { and } \\
\text { reenergizes } \\
\text { after } 1 \text { to } 8 \\
\text { cycles after } \\
\text { event. }\end{array}$ & \multicolumn{4}{|c|}{$\begin{array}{l}\text { EMS drops out } 2 \text { seconds after event below } 65 \% \text { V, but will } \\
\text { automatically restart. } 270 \text { to } 300 \text { seconds first compressor, } 150 \text { to } 240 \\
\text { second interstaging delay for each additional compressor if } \\
\text { applicable. } \\
\text { Contactors operate through voltage variance, but drop out when EMS } \\
\text { drops offline } 2 \text { seconds after event. Or, if the voltage dips below } 50 \% \\
\text { V, the contactor will drop and reenergize } 1 \text { to } 8 \text { cycles after event, } \\
\text { and then drop out again when the EMS drops } 2 \text { seconds later. } \\
\text { Possible thermal trip if the voltage variance is longer than } 4 \text { to } 5 \\
\text { seconds and above } 65 \% \text { V. }\end{array}$} \\
\hline $\begin{array}{l}\text { Fan powered } \\
\text { VAVs }\end{array}$ & $\begin{array}{l}\text { 1-ph } \\
\text { fractional } \\
\text { fan motors }\end{array}$ & $\begin{array}{l}\text { Fuse and } \\
\text { thermal }\end{array}$ & $\begin{array}{l}\text { EMS with } \\
\text { contactor }\end{array}$ & $\begin{array}{l}\text { EMS } \\
\text { remains in } \\
\text { control. } \\
\text { Contactor } \\
\text { drops out at } \\
50 \% \mathrm{~V} \text { and } \\
\text { reenergizes } \\
\text { after } 1 \text { to } 8 \\
\text { cycles after } \\
\text { event. }\end{array}$ & \multicolumn{4}{|c|}{$\begin{array}{l}\text { EMS drops out } 2 \text { seconds after event below } 65 \% \text { V, but will } \\
\text { automatically restart. Fan starts within } 5 \text { seconds. } \\
\text { Contactors operate through voltage variance, but drop out when EMS } \\
\text { drops offline } 2 \text { seconds after event. Or, if the voltage dips below } 50 \% \\
\text { V, the contactor will drop and re-energize } 1 \text { to } 8 \text { cycles after event, } \\
\text { and then drop out again when the EMS drops } 2 \text { seconds later. } \\
\text { Possible thermal trip if the voltage variance is longer than } 4 \text { to } 5 \\
\text { seconds and above } 65 \% \mathrm{~V} \text {. }\end{array}$} \\
\hline $\begin{array}{l}\text { Fan coil } \\
\text { units }\end{array}$ & $\begin{array}{l}\text { 1-ph } \\
\text { fractional } \\
\text { fan motors }\end{array}$ & Thermal & $\begin{array}{l}\text { EMS with } \\
\text { contactor }\end{array}$ & $\begin{array}{l}\begin{array}{l}\text { EMS } \\
\text { remains in } \\
\text { control. }\end{array} \\
\text { Contactor } \\
\text { drops out at } \\
50 \% \mathrm{~V} \text { and } \\
\text { reenergizes } \\
\text { after } 1 \text { to } 8 \\
\text { cycles after } \\
\text { event. }\end{array}$ & \multicolumn{4}{|c|}{$\begin{array}{l}\text { EMS drops out } 2 \text { seconds after event below } 65 \% \mathrm{~V} \text {, but will } \\
\text { automatically restart. Fan starts within } 5 \text { seconds, } \\
\text { Contactors operate through voltage variance, but drop out when EMS } \\
\text { drops offline } 2 \text { seconds after event. Or, if the voltage dips below } 50 \% \\
\text { V, the contactor will drop and re-energize } 1 \text { to } 8 \text { cycles after event, } \\
\text { and then drop out again when the EMS drops } 2 \text { seconds later. } \\
\text { Possible thermal trip if the voltage variance is longer than } 4 \text { to } 5 \\
\text { seconds and above } 65 \% \mathrm{~V} \text {. }\end{array}$} \\
\hline $\begin{array}{l}\text { Building } \\
\text { general } \\
\text { exhaust fans }\end{array}$ & $\begin{array}{l}1 \text { and 3-ph } \\
\text { fan motors }\end{array}$ & $\begin{array}{l}\text { Over voltage, } \\
\text { phase } \\
\text { imbalance, } \\
\text { over current, } \\
\text { or fuse and } \\
\text { thermal }\end{array}$ & $\begin{array}{l}\text { EMS with } \\
\text { VFD or } \\
\text { line voltage } \\
\text { thermostats } \\
\text { / or manual } \\
\text { start/stop } \\
\text { switch }\end{array}$ & $\begin{array}{l}\text { EMS } \\
\text { remains in } \\
\text { control. } \\
\text { Contactor } \\
\text { drops out at } \\
50 \% \mathrm{~V} \text { and } \\
\text { reenergizes } \\
\text { after } 1 \text { to } 8 \\
\text { cycles after } \\
\text { event. }\end{array}$ & \multicolumn{4}{|c|}{$\begin{array}{l}\text { EMS and VFD drop out } 2 \text { seconds after event below } 65 \% \text { V, but will } \\
\text { automatically restart. First fan starts within } 5 \text { seconds, second fan if } \\
\text { applicable restarts at } 30 \text { seconds. } \\
\text { Contactors operate through voltage variance, but drop out when EMS } \\
\text { drops offline } 2 \text { seconds after event. Or, if the voltage dips below } 50 \% \\
\text { V, the contactor will drop and re-energize } 1 \text { to } 8 \text { cycles after event, } \\
\text { and then drop out again when the EMS drops } 2 \text { seconds later }\end{array}$} \\
\hline
\end{tabular}




\begin{tabular}{|c|c|c|c|c|c|c|c|c|}
\hline Equipment & Motors & Protection & Controls & 5 cycle & 10 cycle & 20 cycle & 2 second & 3 minute \\
\hline \multirow[t]{2}{*}{$\begin{array}{l}\text { CRAC } \\
\text { Computer } \\
\text { Room Air } \\
\text { Conditioner }\end{array}$} & $\begin{array}{l}\text { 1-ph or } \\
\text { 3-ph } \\
\text { compressor }\end{array}$ & $\begin{array}{l}\text { Over voltage, } \\
\text { phase } \\
\text { imbalance, } \\
\text { over current, } \\
\text { or fuse and } \\
\text { thermal }\end{array}$ & \multirow[t]{2}{*}{$\begin{array}{l}\text { Manufactur } \\
\text { er Solid- } \\
\text { state with } \\
\text { contactor / } \\
\text { or low } \\
\text { voltage } \\
\text { standalone } \\
\text { thermostats }\end{array}$} & \multirow{2}{*}{$\begin{array}{l}\text { Manufacture } \\
\text { r solid-state } \\
\text { control } \\
\text { board } \\
\text { remains in } \\
\text { control. } \\
\text { Contactor } \\
\text { drops out at } \\
50 \% \text { V and } \\
\text { reenergizes } \\
\text { after } 1 \text { to } 8 \\
\text { cycles after } \\
\text { event. }\end{array}$} & \multicolumn{4}{|c|}{$\begin{array}{l}\text { Manufacturer solid-state control board drops out below } 65 \% \mathrm{~V} \text {, but } \\
\text { will automatically restart. } 270 \text { to } 300 \text { seconds first compressor, } 150 \\
\text { to } 240 \text { second interstaging delay for each additional compressor if } \\
\text { applicable. } \\
\text { Possible thermal trip if the voltage variance is longer than } 3 \text { to } 4 \\
\text { seconds and above } 65 \% \mathrm{~V} \text {. }\end{array}$} \\
\hline & $\begin{array}{l}1 \text {-ph or 3- } \\
\text { ph fan } \\
\text { motor }\end{array}$ & $\begin{array}{l}\text { Over voltage, } \\
\text { phase } \\
\text { imbalance, } \\
\text { over current, } \\
\text { or fuse and } \\
\text { thermal }\end{array}$ & & & \multicolumn{4}{|c|}{$\begin{array}{l}\text { Manufacturer solid-state control board drops out } 2 \text { seconds after } \\
\text { event below } 65 \% \mathrm{~V} \text {, but will automatically restart. Fan starts within } 5 \\
\text { seconds } \\
\text { Possible thermal trip if the voltage variance is longer than } 3 \text { to } 4 \\
\text { seconds and above } 65 \% \mathrm{~V} \text {. }\end{array}$} \\
\hline \multirow[t]{2}{*}{ Boilers } & $\begin{array}{l}\text { 1-ph } \\
\text { induced } \\
\text { draft motor }\end{array}$ & $\begin{array}{l}\text { Fuse and } \\
\text { thermal }\end{array}$ & $\begin{array}{l}\text { Manufactur } \\
\text { er solid- } \\
\text { state with } \\
\text { contactor }\end{array}$ & $\begin{array}{l}\text { Control } \\
\text { Board } \\
\text { remains in } \\
\text { control. } \\
\text { Contactor } \\
\text { drops out at } \\
50 \% \text { V and } \\
\text { reenergizes } \\
\text { after } 1 \text { to } 8 \\
\text { cycles after } \\
\text { event. }\end{array}$ & \multicolumn{4}{|c|}{$\begin{array}{l}\text { Manufacturer solid-state control board drops out below } 65 \% \mathrm{~V} \text {, but } \\
\text { will automatically restart. } 120 \text { seconds first boiler, } 240 \text { seconds } \\
\text { interstaging delay for each additional boiler if applicable. } \\
\text { Possible thermal trip if the voltage variance is longer than } 3 \text { to } 4 \\
\text { seconds and above } 65 \% \mathrm{~V} \text {. }\end{array}$} \\
\hline & $\begin{array}{l}\text { 3-ph } \\
\text { pump } \\
\text { motor }\end{array}$ & $\begin{array}{l}\text { Over voltage, } \\
\text { phase } \\
\text { imbalance, } \\
\text { over current, } \\
\text { and current } \\
\text { limiting }\end{array}$ & $\begin{array}{l}\text { EMS with } \\
\text { VFD }\end{array}$ & $\begin{array}{l}\text { EMS and } \\
\text { VFD remain } \\
\text { in control. }\end{array}$ & \multicolumn{4}{|c|}{$\begin{array}{l}\text { EMS and VFD operate through event, then drop out } 2 \text { seconds after } \\
\text { event below } 65 \% \text { V, but will automatically restart. Pump starts } \\
\text { within } 90 \text { seconds. }\end{array}$} \\
\hline $\begin{array}{l}\text { Domestic hot } \\
\text { water pumps }\end{array}$ & $\begin{array}{l}\text { 1-ph } \\
\text { fractional } \\
\text { motor }\end{array}$ & $\begin{array}{l}\text { Circuit } \\
\text { breaker and } \\
\text { thermal }\end{array}$ & $\begin{array}{l}\text { EMS with } \\
\text { relay / or } \\
\text { Manual } \\
\text { switch }\end{array}$ & $\begin{array}{l}\text { EMS } \\
\text { remains in } \\
\text { control. } \\
\text { Contactor } \\
\text { drops out at } \\
50 \% \text { V and } \\
\text { reenergizes } \\
\text { after } 1 \text { to } 8 \\
\text { cycles after } \\
\text { event. }\end{array}$ & \multicolumn{4}{|c|}{$\begin{array}{l}\text { EMS drops out } 2 \text { seconds after event below } 65 \% \mathrm{~V} \text {, but will } \\
\text { automatically restart within } 10 \text { seconds. } \\
\text { Contactors operate through voltage variance, but drop out when EMS } \\
\text { drops offline } 2 \text { seconds after event. Or, if the voltage dips below } 50 \% \\
\text { V, the contactor will drop and reenergize } 1 \text { to } 8 \text { cycles after event, } \\
\text { and then drop out again when the EMS drops } 2 \text { seconds later. } \\
\text { Possible thermal trip if the voltage variance is longer than } 2 \text { to } 4 \\
\text { seconds and above } 65 \% \text { to } 50 \% \mathrm{~V} \text {. }\end{array}$} \\
\hline $\begin{array}{l}\text { Other plug } \\
\text { loads }\end{array}$ & \multicolumn{8}{|c|}{$\begin{array}{l}\text { Elevators, computers, includes small delis and vending equipment, ATMs, telecommunications equipment, drop out at 50\%, and } \\
\text { computer based loads will go through a reboot sequence on voltage recovery. }\end{array}$} \\
\hline
\end{tabular}


Office Building Load Table

Square feet: 100,000 to $1,000,000$

Voltages between $75 \%$ and $50 \%$ of nominal

\begin{tabular}{|c|c|c|c|c|c|c|c|c|}
\hline Equipment & Motors & Protection & Controls & 5 cycle & 10 cycle & 20 cycle & 2 second & 3 minute \\
\hline AHUs & $\begin{array}{l}\text { 3-ph fan } \\
\text { motors }\end{array}$ & $\begin{array}{l}\text { Over voltage, } \\
\text { phase } \\
\text { imbalance, } \\
\text { over current }\end{array}$ & $\begin{array}{l}\text { EMS with } \\
\text { VFD }\end{array}$ & $\begin{array}{l}\text { EMS remains } \\
\text { in control. }\end{array}$ & \multicolumn{4}{|c|}{$\begin{array}{l}\text { EMS and VFD operate through event, then drop out } 2 \text { seconds } \\
\text { after event below } 65 \% \mathrm{~V} \text {, but will automatically restart. First fan } \\
\text { starts within } 5 \text { seconds, second fan if applicable restarts at } 30 \\
\text { seconds. }\end{array}$} \\
\hline $\begin{array}{l}\text { Fan powered } \\
\text { VAVs }\end{array}$ & $\begin{array}{l}1-\mathrm{ph} \\
\text { fractional } \\
\text { fan motors }\end{array}$ & $\begin{array}{l}\text { Fuse and } \\
\text { thermal }\end{array}$ & $\begin{array}{l}\text { EMS with } \\
\text { contactor }\end{array}$ & $\begin{array}{l}\text { EMS remains } \\
\text { in control. } \\
\text { Contactor } \\
\text { drops out at } \\
50 \% \mathrm{~V} \text { and } \\
\text { reenergizes } \\
\text { after } 1 \text { to } 8 \\
\text { cycles after } \\
\text { event. }\end{array}$ & \multicolumn{4}{|c|}{$\begin{array}{l}\text { EMS drops out } 2 \text { seconds after event below } 65 \% \text { V, but will } \\
\text { automatically restart. Fan starts within } 5 \text { seconds. } \\
\text { Contactors operate through voltage variance, but drop out when } \\
\text { EMS drops offline } 2 \text { seconds after event. Or, if the voltage dips } \\
\text { below } 50 \% \mathrm{~V} \text {, the contactor will drop and reenergize } 1 \text { to } 8 \\
\text { cycles after event, and then drop out again when the EMS drops } \\
2 \text { seconds later. } \\
\text { Possible thermal trip if the voltage variance is longer than } 4 \text { to } 5 \\
\text { seconds and above } 65 \% \mathrm{~V} \text {. }\end{array}$} \\
\hline $\begin{array}{l}\text { (DOAS) } \\
\text { dedicated } \\
\text { outside air } \\
\text { system }\end{array}$ & $\begin{array}{l}\text { 3-ph } \\
\text { fan motors }\end{array}$ & $\begin{array}{l}\text { Over voltage, } \\
\text { phase } \\
\text { imbalance, } \\
\text { over current }\end{array}$ & $\begin{array}{l}\text { EMS with } \\
\text { VFD }\end{array}$ & $\begin{array}{l}\text { EMS remains } \\
\text { in control. }\end{array}$ & \multicolumn{4}{|c|}{$\begin{array}{l}\text { EMS and VFD operate through event, then drop out } 2 \text { seconds } \\
\text { after event below } 65 \% \mathrm{~V} \text {, but will automatically restart. First fan } \\
\text { starts within } 5 \text { seconds, second fan if applicable restarts at } 30 \\
\text { seconds. }\end{array}$} \\
\hline \multirow[t]{2}{*}{ Chillers } & $\begin{array}{l}\text { 3-ph } \\
\text { compressor } \\
\text { motors }\end{array}$ & $\begin{array}{l}\text { Over voltage, } \\
\text { phase } \\
\text { imbalance, } \\
\text { over current }\end{array}$ & $\begin{array}{l}\text { Manufactur } \\
\text { er solid- } \\
\text { state } \\
\text { controller } \\
\text { tied into } \\
\text { EMS }\end{array}$ & $\begin{array}{l}\text { Control board } \\
\text { remains in } \\
\text { control. } \\
\text { Contactor } \\
\text { drops out at } \\
50 \% \text { V and } \\
\text { reenergizes } \\
\text { after } 1 \text { to } 8 \\
\text { cycles after } \\
\text { event. }\end{array}$ & \multicolumn{4}{|c|}{$\begin{array}{l}\text { Manufacturer solid-state control board drops out below } 65 \% \mathrm{~V} \text {, } \\
\text { but will automatically restart. } 300 \text { to } 500 \text { seconds first chiller, } \\
600 \text {-second interstaging delay for each additional chiller if } \\
\text { applicable. }\end{array}$} \\
\hline & $\begin{array}{l}\text { 3-ph } \\
\text { pump } \\
\text { motors }\end{array}$ & $\begin{array}{l}\text { Over voltage, } \\
\text { phase } \\
\text { imbalance, } \\
\text { over current }\end{array}$ & $\begin{array}{l}\text { EMS with } \\
\text { VFD }\end{array}$ & $\begin{array}{l}\text { EMS and } \\
\text { VFD remain } \\
\text { in control. }\end{array}$ & \multicolumn{4}{|c|}{$\begin{array}{l}\text { EMS and VFD operate through event, then drop out } 2 \text { seconds } \\
\text { after event below } 65 \% \mathrm{~V} \text {, but will automatically restart. Pump } \\
\text { soft starts within } 90 \text { seconds. }\end{array}$} \\
\hline \multirow[t]{2}{*}{ Boilers } & $\begin{array}{l}\text { 1-ph } \\
\text { induced } \\
\text { draft motor }\end{array}$ & $\begin{array}{l}\text { Fuse and } \\
\text { thermal }\end{array}$ & $\begin{array}{l}\text { Manufactur } \\
\text { er solid- } \\
\text { state with } \\
\text { contactor, } \\
\text { EMS }\end{array}$ & $\begin{array}{l}\text { Control board } \\
\text { remains in } \\
\text { control. } \\
\text { Contactor } \\
\text { drops out at } \\
50 \% \mathrm{~V} \text { and } \\
\text { reenergizes } \\
\text { after } 1 \text { to } 8 \\
\text { cycles after } \\
\text { event. }\end{array}$ & \multicolumn{4}{|c|}{$\begin{array}{l}\text { Manufacturer solid-state control board drops out below } 65 \% \mathrm{~V} \text {, } \\
\text { but will automatically restart. } 120 \text { seconds first boiler, } 240- \\
\text { second interstaging delay for each additional boiler if applicable. } \\
\text { Possible thermal trip if the voltage variance is long enough and } \\
\text { above } 65 \% \text { for } 2 \text { to } 3 \text { seconds. }\end{array}$} \\
\hline & $\begin{array}{l}\text { 3-ph } \\
\text { motors }\end{array}$ & $\begin{array}{l}\text { Over voltage, } \\
\text { phase } \\
\text { imbalance, } \\
\text { over current, } \\
\text { and current } \\
\text { limiting }\end{array}$ & $\begin{array}{l}\text { EMS with } \\
\text { VFD }\end{array}$ & $\begin{array}{l}\text { EMS and } \\
\text { VFD remain } \\
\text { in control. }\end{array}$ & \multicolumn{4}{|c|}{$\begin{array}{l}\text { EMS and VFD operate through event, then drop out } 2 \text { seconds } \\
\text { after event below } 65 \% \text { V, but will automatically restart. Pump } \\
\text { starts within } 90 \text { seconds. }\end{array}$} \\
\hline $\begin{array}{l}\text { Cooling } \\
\text { towers }\end{array}$ & $\begin{array}{l}\text { 3-ph } \\
\text { fan motor }\end{array}$ & $\begin{array}{l}\text { Over voltage, } \\
\text { phase } \\
\text { imbalance, } \\
\text { over current, }\end{array}$ & $\begin{array}{l}\text { EMS with } \\
\text { VFD }\end{array}$ & $\begin{array}{l}\text { EMS and } \\
\text { VFD remain } \\
\text { in control. }\end{array}$ & \multicolumn{4}{|c|}{$\begin{array}{l}\text { EMS and VFD operate through event, then drop out } 2 \text { seconds } \\
\text { after event below } 65 \% \mathrm{~V} \text {, but will automatically restart. First fan } \\
\text { starts within } 5 \text { seconds, second fan if applicable restarts at } 30 \\
\text { seconds. }\end{array}$} \\
\hline
\end{tabular}




\begin{tabular}{|c|c|c|c|c|c|c|c|c|}
\hline Equipment & Motors & Protection & Controls & 5 cycle & 10 cycle & 20 cycle & 2 second & 3 minute \\
\hline & & $\begin{array}{l}\text { and current } \\
\text { limiting }\end{array}$ & & & & & & \\
\hline $\begin{array}{l}\text { Fan coil } \\
\text { units }\end{array}$ & $\begin{array}{l}\text { 1-ph } \\
\text { fractional } \\
\text { fan motors }\end{array}$ & Thermal & $\begin{array}{l}\text { EMS with } \\
\text { contactor }\end{array}$ & $\begin{array}{l}\text { EMS remains } \\
\text { in control. } \\
\text { Contactor } \\
\text { drops out at } \\
50 \% \text { V and } \\
\text { reenergizes } \\
\text { after } 1 \text { to } 8 \\
\text { cycles after } \\
\text { event. }\end{array}$ & $\begin{array}{l}\text { EMS drops } \\
\text { automaticall } \\
\text { Contactors o } \\
\text { EMS drops } \\
\text { below } 50 \% \\
\text { cycles after } \\
2 \text { seconds la } \\
\text { Possible the } \\
\text { seconds and }\end{array}$ & $\begin{array}{l}\text { seconds aft } \\
\text { tart. Fan sta } \\
\text { te through v } \\
\text { e } 2 \text { seconds } \\
\text { e contactor } \\
\text { t, and then d } \\
\text { trip if the vc } \\
\text { ve } 65 \% \mathrm{~V} \text {. }\end{array}$ & $\begin{array}{l}\text { ent below } 65 \\
\text { thin } 5 \text { secon } \\
\text { e variance, } \\
\text { event. Or, i } \\
\text { rop and reer } \\
\text { ut again wh } \\
\text { variance is }\end{array}$ & $\begin{array}{l}\text {, but will } \\
\text { rop out when } \\
\text { voltage dips } \\
\text { ze } 1 \text { to } 8 \\
\text { e EMS drops } \\
\text { er than } 4 \text { to } 5\end{array}$ \\
\hline $\begin{array}{l}\text { Building } \\
\text { general } \\
\text { exhaust fans }\end{array}$ & $\begin{array}{l}1 \text { and 3-ph } \\
\text { fan motors }\end{array}$ & $\begin{array}{l}\text { Over voltage, } \\
\text { phase } \\
\text { imbalance, } \\
\text { over current, } \\
\text { or fuse and } \\
\text { thermal }\end{array}$ & $\begin{array}{l}\text { EMS with } \\
\text { VFD or } \\
\text { line voltage } \\
\text { thermostats } \\
\text { / or manual } \\
\text { start/stop } \\
\text { switch }\end{array}$ & $\begin{array}{l}\text { EMS remains } \\
\text { in control. } \\
\text { Contactor } \\
\text { drops out at } \\
50 \% \mathrm{~V} \text { and } \\
\text { reenergizes } \\
\text { after } 1 \text { to } 8 \\
\text { cycles after } \\
\text { event. }\end{array}$ & $\begin{array}{l}\text { EMS and Vl } \\
\text { but will auto } \\
\text { second fan i } \\
\text { Contactors o } \\
\text { EMS drops } \\
\text { below } 50 \% \\
\text { cycles after } \\
2 \text { seconds la }\end{array}$ & $\begin{array}{l}\text { rops out } 2 \mathrm{se} \\
\text { cally restart. } \\
\text { licable resta } \\
\text { te through v } \\
\text { e } 2 \text { seconds } \\
\text { e contactor } \\
\text { t, and then d }\end{array}$ & $\begin{array}{l}\text { S after even } \\
\text { fan starts } \mathrm{n} \\
30 \text { seconds. } \\
\text { variance, } \\
\text { event. Or, i } \\
\text { rop and reer } \\
\text { ut again wh }\end{array}$ & $\begin{array}{l}\text { w } 65 \% \text { V, } \\
5 \text { seconds, } \\
\text { rop out when } \\
\text { voltage dips } \\
\text { ze } 1 \text { to } 8 \\
\text { e EMS drops }\end{array}$ \\
\hline \multirow[t]{2}{*}{ CRAC } & $\begin{array}{l}\text { 1-ph or 3-ph } \\
\text { compressor }\end{array}$ & $\begin{array}{l}\text { Over voltage, } \\
\text { phase } \\
\text { imbalance, } \\
\text { over current, } \\
\text { or fuse and } \\
\text { thermal }\end{array}$ & \multirow{2}{*}{$\begin{array}{l}\text { Manu- } \\
\text { facturer } \\
\text { solid-state } \\
\text { with } \\
\text { contactor / } \\
\text { or low } \\
\text { voltage } \\
\text { standalone } \\
\text { thermostats }\end{array}$} & \multirow{2}{*}{$\begin{array}{l}\text { Manufacturer } \\
\text { solid-state } \\
\text { control board } \\
\text { remains in } \\
\text { control. } \\
\text { Contactor } \\
\text { drops out at } \\
50 \% \text { V and } \\
\text { reenergizes } \\
\text { after } 1 \text { to } 8 \\
\text { cycles after } \\
\text { event. }\end{array}$} & \multicolumn{4}{|c|}{$\begin{array}{l}\text { Manufacturer solid-state control board drops out below } 65 \% \mathrm{~V} \text {, } \\
\text { but will automatically restart. } 270 \text { to } 300 \text { seconds first } \\
\text { compressor, } 150 \text { to } 240 \text { seconds interstaging delay for each } \\
\text { additional compressor if applicable. } \\
\text { Possible thermal trip if the voltage variance is longer than } 4 \text { to } 5 \\
\text { seconds and above } 65 \% \mathrm{~V} \text {. }\end{array}$} \\
\hline & $\begin{array}{l}\text { 1-ph or 3-ph } \\
\text { fan motor }\end{array}$ & $\begin{array}{l}\text { Over voltage, } \\
\text { phase } \\
\text { imbalance, } \\
\text { over current, } \\
\text { or fuse and } \\
\text { thermal }\end{array}$ & & & \multicolumn{4}{|c|}{$\begin{array}{l}\text { Manufacturer solid-state control board drops out } 2 \text { seconds after } \\
\text { event below } 65 \% \mathrm{~V} \text {, but will automatically restart. Fan starts } \\
\text { within } 5 \text { seconds. } \\
\text { Possible thermal trip if the voltage variance is longer than } 4 \text { to } 5 \\
\text { seconds and above } 65 \% \mathrm{~V} \text {. }\end{array}$} \\
\hline $\begin{array}{l}\text { Domestic } \\
\text { cold water } \\
\text { pumps }\end{array}$ & $\begin{array}{l}\text { 3-ph } \\
\text { pump motor }\end{array}$ & $\begin{array}{l}\text { Over voltage, } \\
\text { phase } \\
\text { imbalance, } \\
\text { over current }\end{array}$ & $\begin{array}{l}\text { Manu- } \\
\text { facturer } \\
\text { solid-state } \\
\text { with VFD }\end{array}$ & $\begin{array}{l}\text { Manufacturer } \\
\text { solid-state } \\
\text { control board } \\
\text { remains in } \\
\text { control. } \\
\text { Contactor } \\
\text { drops out at } \\
50 \% \text { V and } \\
\text { reenergizes } \\
\text { after } 1 \text { to } 8 \\
\text { cycles after } \\
\text { event. }\end{array}$ & \multicolumn{4}{|c|}{$\begin{array}{l}\text { Manufacturer solid-state control board drops out } 2 \text { seconds after } \\
\text { event below } 65 \% \mathrm{~V} \text {, but will automatically restart. Pumps soft } \\
\text { start within } 5 \text { seconds. }\end{array}$} \\
\hline $\begin{array}{l}\text { Domestic } \\
\text { hot water } \\
\text { pumps }\end{array}$ & $\begin{array}{l}\text { 1-ph } \\
\text { fractional } \\
\text { motor }\end{array}$ & $\begin{array}{l}\text { Circuit } \\
\text { breaker and } \\
\text { thermal }\end{array}$ & $\begin{array}{l}\text { EMS with } \\
\text { relay / or } \\
\text { manual } \\
\text { switch }\end{array}$ & $\begin{array}{l}\text { EMS remains } \\
\text { in control. } \\
\text { Contactor } \\
\text { drops out at } \\
50 \% \mathrm{~V} \text { and } \\
\text { reenergizes } \\
\text { after } 1 \text { to } 8 \\
\text { cycles after } \\
\text { event. }\end{array}$ & \multicolumn{4}{|c|}{$\begin{array}{l}\text { EMS drops out } 2 \text { seconds after event below } 65 \% \mathrm{~V} \text {, but will } \\
\text { automatically restart within } 10 \text { seconds. } \\
\text { Contactors operate through voltage variance, but drop out when } \\
\text { EMS drops offline } 2 \text { seconds after event. Or, if the voltage dips } \\
\text { below } 50 \% \mathrm{~V} \text {, the contactor will drop and reenergizes } 1 \text { to } 8 \\
\text { cycles after event, and then drop out again when the EMS drops } \\
2 \text { seconds later. } \\
\text { Possible thermal trip if the voltage variance is longer than } 3 \text { to } 4 \\
\text { seconds and above } 65 \% \mathrm{~V} \text {. }\end{array}$} \\
\hline
\end{tabular}




\begin{tabular}{|c|c|c|c|c|c|c|c|c|}
\hline Equipment & Motors & Protection & Controls & 5 cycle & 10 cycle & 20 cycle & 2 second & 3 minute \\
\hline $\begin{array}{l}\text { Sewage } \\
\text { rejection }\end{array}$ & $\begin{array}{l}\text { 3-ph } \\
\text { pump motor }\end{array}$ & $\begin{array}{l}\text { Fused with } \\
\text { current } \\
\text { overload }\end{array}$ & $\begin{array}{l}\text { Line } \\
\text { voltage } \\
\text { mag-starter } \\
\text { start/stop } \\
\text { level switch }\end{array}$ & \multicolumn{3}{|c|}{$\begin{array}{l}\text { Contactor drops out at } 50 \% \mathrm{~V} \text {. Reenergizes } \\
\text { after } 1 \text { to } 8 \text { cycles after event. }\end{array}$} & \multicolumn{2}{|c|}{$\begin{array}{l}\text { Contactor drops out at } 50 \% \mathrm{~V} \text {. } \\
\text { Reenergizes after } 1 \text { to } 8 \text { cycles } \\
\text { after event. } \\
\text { Possible overload trip if the } \\
\text { voltage variance is long enough } \\
\text { and above } 50 \% \mathrm{~V} \text {. }\end{array}$} \\
\hline Other loads & $\begin{array}{l}\text { levators, } \\
\text { omputer b }\end{array}$ & $\begin{array}{l}\text { ters, inclu } \\
\text { pads will }\end{array}$ & $\begin{array}{l}\text { all delis a } \\
\text { ugh a rebc }\end{array}$ & $\begin{array}{l}\text { ending eq } \\
\text { equence o }\end{array}$ & $\begin{array}{l}\text { ATMs, t } \\
\text { recovery }\end{array}$ & ica & & $\%$, and \\
\hline
\end{tabular}


Retail Building Load Table

Square feet: 5,000 and under

Voltage between $75 \%$ and $50 \%$ of nominal

\begin{tabular}{|c|c|c|c|c|c|c|c|c|}
\hline Equipment & Motors & Protection & Controls & 5 cycle & 10 cycle & 20 cycle & 2 second & 3 minute \\
\hline \multirow[t]{3}{*}{ RTUs } & $\begin{array}{l}\text { 3-ph indoor } \\
\text { fan motors }\end{array}$ & $\begin{array}{l}\text { Fused } \\
\text { possibly } \\
\text { overload } \\
\text { relays }\end{array}$ & $\begin{array}{l}\text { Low voltage } \\
\text { thermostat } \\
\text { with } \\
\text { contactor }\end{array}$ & \multirow{3}{*}{$\begin{array}{l}\text { Thermostat } \\
\text { remains in } \\
\text { control. } \\
\text { Contactor } \\
\text { drops out at } \\
50 \% \text { V and } \\
\text { reenergizes } \\
\text { after } 1 \text { to } 8 \\
\text { cycles after } \\
\text { event. }\end{array}$} & \multicolumn{4}{|c|}{$\begin{array}{l}\text { Thermostat will drop out below } 65 \% \mathrm{~V} \text {, but will automatically } \\
\text { restart. Fan starts within } 30 \text { seconds. } \\
\text { Possible thermal trip if the voltage variance is longer than } 4 \text { to } 5 \\
\text { seconds and above } 65 \% \mathrm{~V} \text {. }\end{array}$} \\
\hline & $\begin{array}{l}\text { 3-ph } \\
\text { compressor } \\
\text { motors }\end{array}$ & $\begin{array}{l}\text { Fuse and } \\
\text { thermal }\end{array}$ & $\begin{array}{l}\text { Low voltage } \\
\text { thermostat } \\
\text { with } \\
\text { contactor }\end{array}$ & & \multicolumn{4}{|c|}{$\begin{array}{l}\text { Thermostat will drop out below } 65 \% \mathrm{~V} \text {, but will automatically } \\
\text { restart the compressor within } 300 \text { seconds. } \\
\text { Possible thermal trip if the voltage variance is longer than } 4 \text { to } 5 \\
\text { seconds and above } 65 \% \mathrm{~V} \text {. }\end{array}$} \\
\hline & $\begin{array}{l}\text { 1-ph } \\
\text { fractional } \\
\text { condenser } \\
\text { and induced } \\
\text { draft motors } \\
\end{array}$ & $\begin{array}{l}\text { Fuse and } \\
\text { thermal }\end{array}$ & $\begin{array}{l}\text { Low voltage } \\
\text { thermostat } \\
\text { with } \\
\text { contactor }\end{array}$ & & \multicolumn{4}{|c|}{$\begin{array}{l}\text { Thermostat will drop out below } 65 \% \mathrm{~V} \text {, but will automatically } \\
\text { restart the fans with the compressor within } 300 \text { seconds. } \\
\text { Possible thermal trip if the voltage variance is longer than } 4 \text { to } 5 \\
\text { seconds and above } 65 \% \mathrm{~V} \text {. }\end{array}$} \\
\hline \multirow[t]{2}{*}{$\begin{array}{l}\text { Walk-in } \\
\text { refrigeration } \\
\text { and freezers }\end{array}$} & $\begin{array}{l}\text { 1-ph or 3-ph } \\
\text { compressors }\end{array}$ & $\begin{array}{l}\text { Fuse and } \\
\text { thermal }\end{array}$ & $\begin{array}{l}\text { Line voltage } \\
\text { with } \\
\text { contactor }\end{array}$ & \multirow{2}{*}{$\begin{array}{l}\text { Contactor } \\
\text { drops out at } \\
50 \% \text { V. } \\
\text { Reenergizes } \\
\text { after } 1 \text { to } 8 \\
\text { cycles after } \\
\text { event. }\end{array}$} & \multirow{2}{*}{\multicolumn{4}{|c|}{$\begin{array}{l}\text { Automatically restart. } 300 \text { seconds for compressor and all } \\
\text { associated 1-phase fans. } \\
\text { Possible thermal trip if the voltage variance is longer than } 2 \text { to } 3 \\
\text { seconds and above } 50 \% \mathrm{~V} \text {. }\end{array}$}} \\
\hline & $\begin{array}{l}1 \text {-ph } \\
\text { fractional } \\
\text { evaporator } \\
\text { and } \\
\text { condenser } \\
\text { fan motors }\end{array}$ & $\begin{array}{l}\text { Fuse and } \\
\text { thermal }\end{array}$ & $\begin{array}{l}\text { Line voltage } \\
\text { with } \\
\text { contactor }\end{array}$ & & & & & \\
\hline Exhaust fans & $\begin{array}{l}\text { 1-ph } \\
\text { fractional } \\
\text { motor }\end{array}$ & $\begin{array}{l}\text { Circuit } \\
\text { breaker and } \\
\text { thermal }\end{array}$ & $\begin{array}{l}\text { Manual } \\
\text { switch }\end{array}$ & \multicolumn{3}{|c|}{$\begin{array}{l}\text { Contactor drops out at } 50 \% \mathrm{~V} \text {. Reenergizes } 1 \text { to } \\
8 \text { cycles after event. }\end{array}$} & \multicolumn{2}{|c|}{$\begin{array}{l}\text { Contactor drops out at } 50 \% \mathrm{~V} \\
\text { Reenergizes after } 1 \text { to } 8 \text { cycles } \\
\text { after event. } \\
\text { Possible Thermal trip if the } \\
\text { voltage variance is long enough } \\
\text { and above } 50 \% \mathrm{~V} \text {. }\end{array}$} \\
\hline $\begin{array}{l}\text { Reach-in } \\
\text { refrigeration } \\
\text { and freezers }\end{array}$ & $\begin{array}{l}\text { 1-ph } \\
\text { fractional } \\
\text { compressor } \\
\text { condenser } \\
\text { and } \\
\text { evaporator } \\
\text { fan motors }\end{array}$ & Thermal & $\begin{array}{l}\text { Solid-state } \\
\text { with relay } \\
\text { from control } \\
\text { board }\end{array}$ & $\begin{array}{l}\text { Motor will } \\
\text { continue to } \\
\text { operate } \\
\text { through } \\
\text { voltage } \\
\text { variance. }\end{array}$ & \multicolumn{4}{|c|}{$\begin{array}{l}\text { The solid-state control board will drop out below } 65 \% \text { V. Manual } \\
\text { reset needed. } \\
\text { Possible thermal trip if the voltage variance is longer than } 4 \text { to } 5 \\
\text { seconds and above } 65 \% \mathrm{~V} \text {. }\end{array}$} \\
\hline $\begin{array}{l}\text { Other plug } \\
\text { loads }\end{array}$ & \multicolumn{8}{|c|}{$\begin{array}{l}\text { Gas pumps, refrigerated display cases, ice bins, TVs and monitors, cooking equipment drop out at 75\% voltage and computer based } \\
\text { loads will restart after reboot sequence. }\end{array}$} \\
\hline
\end{tabular}


Retail Building Load Table

Square feet: 15,000 to 40,000

Voltages between $75 \%$ and $50 \%$ of nominal

\begin{tabular}{|c|c|c|c|c|c|c|c|c|}
\hline Equipment & Motors & Protection & Controls & 5 cycle & 10 cycle & 20 cycle & 2 second & 3 minute \\
\hline \multirow[t]{3}{*}{ RTUs } & $\begin{array}{l}\text { 3-ph indoor } \\
\text { fan motors }\end{array}$ & $\begin{array}{l}\text { Fused } \\
\text { possibly } \\
\text { overload } \\
\text { relays }\end{array}$ & \multirow{3}{*}{$\begin{array}{l}\text { EMS } \\
\text { or } \\
\text { Manufacturer } \\
\text { solid state } \\
\text { tied into ems } \\
\text { with } \\
\text { contactors } \\
\text { or } \\
\text { Low voltage } \\
\text { standalone } \\
\text { thermostats }\end{array}$} & \multirow{3}{*}{$\begin{array}{l}\text { Thermostat } \\
\text { remains in } \\
\text { control. } \\
\text { Contactor } \\
\text { drops out at } \\
50 \% \text { V and } \\
\text { reenergizes } \\
\text { after } 1 \text { to } 8 \\
\text { cycles after } \\
\text { event. }\end{array}$} & \multicolumn{4}{|c|}{$\begin{array}{l}\text { EMS or thermostat will drop out below } 65 \% \mathrm{~V} \text {, but will } \\
\text { automatically restart. Fan starts within } 30 \text { seconds. } \\
\text { Possible thermal trip if the voltage variance is longer than } 4 \text { to } 5 \\
\text { seconds and above } 65 \% \mathrm{~V} \text {. }\end{array}$} \\
\hline & $\begin{array}{l}\text { 3-ph } \\
\text { compressor } \\
\text { motors }\end{array}$ & $\begin{array}{l}\text { Fuse and } \\
\text { thermal }\end{array}$ & & & \multicolumn{4}{|c|}{$\begin{array}{l}\text { EMS or thermostat will drop out below } 65 \% \mathrm{~V} \text {, but will } \\
\text { automatically restart the compressor within } 300 \text { seconds. } \\
\text { Possible thermal trip if the voltage variance is longer than } 4 \text { to } 5 \\
\text { seconds and above } 65 \% \mathrm{~V} \text {. }\end{array}$} \\
\hline & $\begin{array}{l}\text { 1-ph } \\
\text { fractional } \\
\text { condenser } \\
\text { and induced } \\
\text { draft motors }\end{array}$ & $\begin{array}{l}\text { Fuse and } \\
\text { thermal }\end{array}$ & & & \multicolumn{4}{|c|}{$\begin{array}{l}\text { EMS or thermostat will drop out below } 65 \% \mathrm{~V} \text {, but will } \\
\text { automatically restart the fans with the compressor within } 300 \\
\text { seconds. } \\
\text { Possible thermal trip if the voltage variance is longer than } 4 \text { to } 5 \\
\text { seconds and above } 65 \% \mathrm{~V} \text {. }\end{array}$} \\
\hline $\begin{array}{l}\text { Exhaust } \\
\text { fans }\end{array}$ & $\begin{array}{l}1 \text {-ph } \\
\text { fractional } \\
\text { motor }\end{array}$ & $\begin{array}{l}\text { Circuit } \\
\text { breaker and } \\
\text { thermal }\end{array}$ & $\begin{array}{l}\text { Manual } \\
\text { switch }\end{array}$ & \multicolumn{3}{|c|}{$\begin{array}{l}\text { Contactor drops out at } 50 \% \mathrm{~V} \text {. } \\
\text { Reenergizes after } 1 \text { to } 8 \text { cycles after event. }\end{array}$} & \multicolumn{2}{|c|}{$\begin{array}{l}\text { Contactor drops out at } 50 \% \mathrm{~V} \text {. } \\
\text { Reenergizes after } 1 \text { to } 8 \text { cycles } \\
\text { after event. } \\
\text { Possible thermal trip if the voltage } \\
\text { variance is long enough and above } \\
50 \% \mathrm{~V} \text {. }\end{array}$} \\
\hline $\begin{array}{l}\text { Other plug } \\
\text { loads }\end{array}$ & \multicolumn{8}{|c|}{$\begin{array}{l}\text { Elevators, computers, refrigeration, cooking includes small delis and vending equipment, ATMs, telecommunications equipment } \\
\text { drop out at } 75 \% \text { voltage, and recover immediately with voltage recovery except for computer-based load with a reboot sequence. }\end{array}$} \\
\hline
\end{tabular}


Retail Building Load Table

Square feet: 15,000 to 100,000

Voltages between $75 \%$ and $50 \%$ of nominal

\begin{tabular}{|c|c|c|c|c|c|c|c|c|}
\hline Equipment & Motors & Protection & Controls & 5 cycle & 10 cycle & 20 cycle & 2 second & 3 minute \\
\hline \multirow[t]{3}{*}{ RTUs } & $\begin{array}{l}\text { 3-ph indoor } \\
\text { fan motors }\end{array}$ & $\begin{array}{l}\text { Fused } \\
\text { possibly } \\
\text { overload } \\
\text { relays }\end{array}$ & \multirow{3}{*}{$\begin{array}{l}\text { EMS } \\
\text { or } \\
\text { Manufacturer solid } \\
\text { state tied into EMS } \\
\text { with contactors } \\
\text { or } \\
\text { Low voltage } \\
\text { standalone } \\
\text { thermostats }\end{array}$} & \multirow{3}{*}{$\begin{array}{l}\text { Thermostat } \\
\text { remains in } \\
\text { control. } \\
\text { Contactor } \\
\text { drops out } \\
\text { at } 50 \% \mathrm{~V} \\
\text { and } \\
\text { reenergizes } \\
\text { after } 1 \text { to } 8 \\
\text { cycles } \\
\text { after event. }\end{array}$} & \multicolumn{4}{|c|}{$\begin{array}{l}\text { EMS or thermostat will drop out below } 65 \% \mathrm{~V} \text {, but will } \\
\text { automatically restart. Fan starts within } 30 \text { seconds. } \\
\text { Possible thermal trip if the voltage variance is longer than } 4-5 \\
\text { seconds and above } 65 \% \mathrm{~V} \text {. }\end{array}$} \\
\hline & $\begin{array}{l}\text { 3-ph } \\
\text { compressor } \\
\text { motors }\end{array}$ & $\begin{array}{l}\text { Fuse and } \\
\text { thermal }\end{array}$ & & & \multicolumn{4}{|c|}{$\begin{array}{l}\text { EMS or thermostat will drop out below } 65 \% \mathrm{~V} \text {, but will } \\
\text { automatically restart the compressor within } 300 \text { seconds. } \\
\text { Possible thermal trip if the voltage variance is longer than } 4-5 \\
\text { seconds and above } 65 \% \mathrm{~V} \text {. }\end{array}$} \\
\hline & $\begin{array}{l}\text { 1-ph } \\
\text { fractional } \\
\text { condenser } \\
\text { and } \\
\text { induced } \\
\text { draft } \\
\text { motors }\end{array}$ & $\begin{array}{l}\text { Fuse and } \\
\text { thermal }\end{array}$ & & & \multicolumn{4}{|c|}{$\begin{array}{l}\text { EMS or thermostat will drop out below } 65 \% \mathrm{~V} \text {, but will } \\
\text { automatically restart the fans with the compressor within } 300 \\
\text { seconds. } \\
\text { Possible thermal trip if the voltage variance is longer than } 4 \\
\text { to } 5 \text { seconds and above } 65 \% \mathrm{~V} \text {. }\end{array}$} \\
\hline $\begin{array}{l}\text { Exhaust } \\
\text { fans }\end{array}$ & $\begin{array}{l}\text { 1-ph } \\
\text { fractional } \\
\text { motor }\end{array}$ & $\begin{array}{l}\text { Circuit } \\
\text { breaker and } \\
\text { thermal }\end{array}$ & Manual switch & \multicolumn{3}{|c|}{$\begin{array}{l}\text { Contactor drops out at } 50 \% \mathrm{~V} \text {. } \\
\text { Reenergizes after } 1 \text { to } 8 \text { cycles after } \\
\text { event. }\end{array}$} & \multicolumn{2}{|c|}{$\begin{array}{l}\text { Contactor drops out at } 50 \% \mathrm{~V} \text {. } \\
\text { Reenergizes after } 1 \text { to } 8 \text { cycles } \\
\text { after event. } \\
\text { Possible thermal trip if the } \\
\text { voltage variance is long enough } \\
\text { and above } 50 \% \text { V. }\end{array}$} \\
\hline $\begin{array}{l}\text { Other plug } \\
\text { loads }\end{array}$ & \multicolumn{8}{|c|}{$\begin{array}{l}\text { Elevators, escalators, computers, refrigeration, vending equipment, ATMs, telecommunications equipment drops out at } 75 \% \text { voltage } \\
\text { and restarts with voltage recovery, except for computer-based, which must go through reboot sequence. }\end{array}$} \\
\hline
\end{tabular}


Warehouse Building Load Table

Square feet: 20,000

Voltages between $75 \%$ and $50 \%$ of nominal

\begin{tabular}{|c|c|c|c|c|c|c|c|c|}
\hline Equipment & Motors & Protection & Controls & 5 cycle & 10 cycle & 20 cycle & 2 second & 3 minute \\
\hline $\begin{array}{l}\text { Gas fired unit } \\
\text { heaters }\end{array}$ & $\begin{array}{l}\text { 1-ph } \\
\text { fractional fan } \\
\text { motors }\end{array}$ & Thermal & $\begin{array}{l}\text { Low voltage } \\
\text { thermostat with } \\
\text { contactor }\end{array}$ & $\begin{array}{l}\text { Thermostat } \\
\text { remains in } \\
\text { control. } \\
\text { Contactor } \\
\text { drops out at } \\
50 \% \text { V and } \\
\text { reenergizes } \\
\text { after } 1 \text { to } 8 \\
\text { cycles after } \\
\text { event. }\end{array}$ & \multicolumn{4}{|c|}{$\begin{array}{l}\text { EMS or Thermostat will drop out below } 65 \% \text { V. but will } \\
\text { automatically restart. Fan starts within } 30 \text { seconds. } \\
\text { Possible thermal trip if the voltage variance is longer than } 4 \\
\text { to } 5 \text { seconds and above } 65 \% \mathrm{~V} \text {. }\end{array}$} \\
\hline Exhaust fans & $\begin{array}{l}\text { 1-ph } \\
\text { fractional } \\
\text { motor }\end{array}$ & Thermal & Manual switch & \multicolumn{3}{|c|}{$\begin{array}{l}\text { Contactor drops out at } 50 \% \mathrm{~V} \text {. } \\
\text { Reenergizes after } 1 \text { to } 8 \text { cycles after } \\
\text { event. }\end{array}$} & \multicolumn{2}{|c|}{$\begin{array}{l}\text { Contactor drops out at } 50 \% \mathrm{~V} \text {. } \\
\text { Reenergizes after } 1 \text { to } 8 \text { cycles } \\
\text { after event. } \\
\text { Possible thermal trip if the } \\
\text { voltage variance is long enough } \\
\text { and above } 50 \% \mathrm{~V} \text {. }\end{array}$} \\
\hline Paddle fans & $\begin{array}{l}1-\mathrm{ph} \\
\text { fractional } \\
\text { motor }\end{array}$ & Thermal & Manual switch & \multicolumn{3}{|c|}{$\begin{array}{l}\text { Contactor drops out at } 50 \% \mathrm{~V} \text {. } \\
\text { Reenergizes after } 1 \text { to } 8 \text { cycles after } \\
\text { event. }\end{array}$} & \multicolumn{2}{|c|}{$\begin{array}{l}\text { Contactor drops out at } 50 \% \mathrm{~V} \text {. } \\
\text { Reenergizes after } 1 \text { to } 8 \text { cycles } \\
\text { after event. } \\
\text { Possible thermal trip if the } \\
\text { voltage variance is long enough } \\
\text { and above } 50 \% \text {. }\end{array}$} \\
\hline $\begin{array}{l}\text { Other plug } \\
\text { loads }\end{array}$ & \multicolumn{8}{|c|}{$\begin{array}{l}\text { Electric forklift charging, TV and monitors, computers, copiers, fax, printers, refrigerators, microwaves, box fans will drop off at } \\
75 \% \text { and restart with voltage recovery. Computer-based loads will go through reboot sequence. }\end{array}$} \\
\hline
\end{tabular}


Other Building Load Table

Voltages between $75 \%$ and $50 \%$ of nominal

\begin{tabular}{|c|c|c|c|c|c|c|c|c|}
\hline Equipment & Motors & Protection & Controls & 5 cycle & 10 cycle & 20 cycle & 2 second & 3 minute \\
\hline \multirow[t]{2}{*}{$\begin{array}{l}\text { Single phase } \\
\text { heat pumps }\end{array}$} & $\begin{array}{l}\text { 1-ph } \\
\text { fractional HP } \\
\text { motors }\end{array}$ & $\begin{array}{l}\text { Fuse and } \\
\text { thermal }\end{array}$ & $\begin{array}{l}\text { Low voltage } \\
\text { thermostat with } \\
\text { contactor }\end{array}$ & $\begin{array}{l}\text { Stall } \\
\text { between } \\
60 \% \text { and } \\
70 \% \text {. } \\
\text { Disconnect } \\
\text { at } 50 \% \text {. Do } \\
\text { not restart } \\
\text { for } 3 \text { to } 5 \\
\text { minutes. } \\
\end{array}$ & \multicolumn{4}{|c|}{$\begin{array}{l}\text { Thermostat will drop out below } 65 \% \mathrm{~V} \text {, but will } \\
\text { automatically restart. Fan starts within } 30 \text { seconds. } \\
\text { Possible thermal trip if the voltage variance is longer than } \\
4 \text { to } 5 \text { seconds and above } 65 \% \mathrm{~V} \text {. }\end{array}$} \\
\hline & $\begin{array}{l}\text { 1-ph } \\
\text { compressor } \\
\text { motor }\end{array}$ & $\begin{array}{l}\text { Fuse and } \\
\text { thermal }\end{array}$ & $\begin{array}{l}\text { Battery backed low } \\
\text { voltage thermostat } \\
\text { with contactor }\end{array}$ & $\begin{array}{l}\text { Drop out at } \\
50 \% \text { V and } \\
\text { restart at } \\
90 \% \mathrm{~V} \text {. }\end{array}$ & \multicolumn{4}{|c|}{$\begin{array}{l}\text { Thermostat will drop out below } 65 \% \mathrm{~V} \text {, but will } \\
\text { automatically restart the compressor within } 300 \text { seconds. } \\
\text { Possible thermal trip if the voltage variance is longer than } \\
4 \text { to } 5 \text { seconds and above } 65 \% \mathrm{~V} \text {. }\end{array}$} \\
\hline \multirow[t]{3}{*}{ RTUs } & $\begin{array}{l}\text { 3-ph indoor } \\
\text { fan motors }\end{array}$ & $\begin{array}{l}\text { Fused } \\
\text { possibly } \\
\text { overload } \\
\text { relays }\end{array}$ & \multirow{3}{*}{$\begin{array}{l}\text { EMS } \\
\text { or Manufacturer } \\
\text { solid state tied into } \\
\text { EMS with } \\
\text { contactors } \\
\text { or } \\
\text { Low voltage } \\
\text { standalone } \\
\text { thermostats }\end{array}$} & \multirow{3}{*}{$\begin{array}{l}\text { Thermostat } \\
\text { remains in } \\
\text { control. } \\
\text { Contactor } \\
\text { drops out at } \\
50 \% \text { V and } \\
\text { reenergizes } \\
\text { after } 1 \text { to } 8 \\
\text { cycles after } \\
\text { event. }\end{array}$} & \multicolumn{4}{|c|}{$\begin{array}{l}\text { Thermostat will drop out below } 65 \% \mathrm{~V} \text {, but will } \\
\text { automatically restart. Fan starts within } 30 \text { seconds. } \\
\text { Possible thermal trip if the voltage variance is longer than } \\
4 \text { to } 5 \text { seconds and above } 65 \% \mathrm{~V} \text {. }\end{array}$} \\
\hline & $\begin{array}{l}\text { 3-ph } \\
\text { compressor } \\
\text { motors }\end{array}$ & $\begin{array}{l}\text { Fuse and } \\
\text { thermal }\end{array}$ & & & \multicolumn{4}{|c|}{$\begin{array}{l}\text { Thermostat will drop out below } 65 \% \mathrm{~V} \text {, but will } \\
\text { automatically restart the compressor within } 300 \text { seconds. } \\
\text { Possible thermal trip if the voltage variance is longer than } \\
4 \text { to } 5 \text { seconds and above } 65 \% \mathrm{~V} \text {. }\end{array}$} \\
\hline & $\begin{array}{l}\text { 1-ph } \\
\text { fractional } \\
\text { condenser and } \\
\text { induced draft } \\
\text { motors }\end{array}$ & $\begin{array}{l}\text { Fuse and } \\
\text { thermal }\end{array}$ & & & \multicolumn{4}{|c|}{$\begin{array}{l}\text { Thermostat will drop out below } 65 \% \mathrm{~V} \text {, but will } \\
\text { automatically restart the fans with the compressor within } \\
300 \text { seconds. } \\
\text { Possible thermal trip if the voltage variance is longer than } \\
4 \text { to } 5 \text { seconds and above } 65 \% \mathrm{~V} \text {. }\end{array}$} \\
\hline $\begin{array}{l}\text { Electric heat } \\
\text { fan motors }\end{array}$ & $\begin{array}{l}\text { 1-ph } \\
\text { fractional fan } \\
\text { motors }\end{array}$ & Thermal & $\begin{array}{l}\text { Low voltage } \\
\text { thermostat with } \\
\text { contactor }\end{array}$ & $\begin{array}{l}\text { Thermostat } \\
\text { remains in } \\
\text { control. } \\
\text { Contactor } \\
\text { drops out at } \\
50 \% \text { V and } \\
\text { reenergizes } \\
\text { after } 1 \text { to } 8 \\
\text { cycles after } \\
\text { event. }\end{array}$ & \multicolumn{4}{|c|}{$\begin{array}{l}\text { EMS or thermostat will drop out below } 65 \% \mathrm{~V} \text {, but will } \\
\text { automatically restart. Fan starts within } 30 \text { seconds. } \\
\text { Possible thermal trip if the voltage variance is longer than } \\
4 \text { to } 5 \text { seconds and above } 65 \% \mathrm{~V} \text {. }\end{array}$} \\
\hline $\begin{array}{l}\text { Misc. plug } \\
\text { loads, } \\
\text { computers, } \\
\text { vending, } \\
\text { ATMs }\end{array}$ & $\begin{array}{l}\text { Exhaust fans } \\
\text { and } \\
\text { compressor } \\
\text { motors, single } \\
\text { phase, } \\
\text { fractional HP } \\
\end{array}$ & $\begin{array}{l}\text { Thermal } \\
\text { and fuse }\end{array}$ & Local relay & \multicolumn{5}{|c|}{$\begin{array}{l}\text { Battery-backed devices remain on. Others drop out at 50\% V. } \\
\text { 1-minute delay on voltage recovery. }\end{array}$} \\
\hline
\end{tabular}





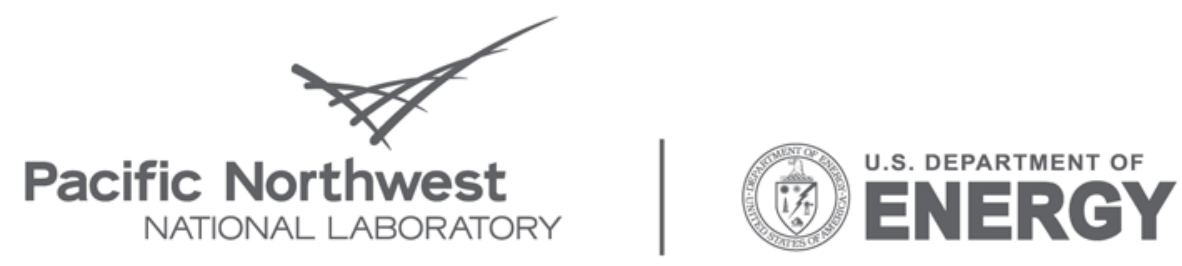

Proudly Operated by Battelle Since 1965

902 Battelle Boulevard

P.O. Box 999

Richland, WA 99352

1-888-375-PNNL (7665)

www.pnl.gov 